\title{
A Consensus Based Decentralized State Estimation for Power Distribution Networks
}

Varun Garaga

Follow this and additional works at: https://researchrepository.wvu.edu/etd

\section{Recommended Citation}

Garaga, Varun, "A Consensus Based Decentralized State Estimation for Power Distribution Networks" (2015). Graduate Theses, Dissertations, and Problem Reports. 5647.

https://researchrepository.wvu.edu/etd/5647

This Thesis is protected by copyright and/or related rights. It has been brought to you by the The Research Repository @ WVU with permission from the rights-holder(s). You are free to use this Thesis in any way that is permitted by the copyright and related rights legislation that applies to your use. For other uses you must obtain permission from the rights-holder(s) directly, unless additional rights are indicated by a Creative Commons license in the record and/ or on the work itself. This Thesis has been accepted for inclusion in WVU Graduate Theses, Dissertations, and Problem Reports collection by an authorized administrator of The Research Repository @ WVU. For more information, please contact researchrepository@mail.wvu.edu. 


\title{
A Consensus Based Decentralized State Estimation for Power Distribution Networks \\ Varun Garaga
}

\author{
Thesis submitted to the \\ Benjamin M. Statler College of Engineering and Mineral Resources \\ at West Virginia University \\ in partial fulfillment of the requirements for the degree of \\ Master of Science \\ in \\ Electrical Engineering
}

Dr. Ali Feliachi, Ph.D., Chair

Dr. Sarika Kushalani Solanki, Ph.D.

Dr. Muhammad Choudhry, Ph.D.

Lane Department of Computer Science and Electrical Engineering

Morgantown, West Virginia

2015

Keywords: State Estimation, Decentralized, Distribution Networks, Consensus and Power Flow

Copyright 2015 Varun Garaga 


\section{ABSTRACT \\ A Consensus Based Decentralized State Estimation for Power Distribution Networks \\ Varun Garaga}

This thesis presents a new Decentralized State Estimation algorithm using agents directed mainly to distribution power systems. This new algorithm solves problems that occur when one tries to estimate the state of the distribution power systems. By various reasons such as high levels of quality of service, automation capabilities and comparatively less size, those problems do not occur so frequently on the transmission systems. A consensus based static state estimation strategy for radial power distribution systems is proposed in this research. This thesis concentrates on the balanced systems.

There are buses acting as agents using which we can evaluate the local estimates of the entire system. Therefore each measurement model reduces to an underdetermined nonlinear system and in radial distribution systems, the state elements associated with an agent may overlap with neighboring agents. We propose a state estimation strategy, which effectively integrates the principles of local consensus and least squares technique and finally provides a decentralized solution to the radial power distribution grid. At the end of the thesis, we present the results of the application of the developed approach to a network based on a modified IEEE 13 bus test system and IEEE 33 bus Test System. The states of these systems are first estimated through centralized approach using least squares technique to compare with the proposed algorithm. 


\section{Acknowledgements}

First of all, I would like to express my most sincere gratitude to my advisor Dr. Ali Feliachi for his continuous support of my Master's study and research, for his patience, motivation, expertise and understanding. His guidance helped me throughout the research period. I would also like to thank my other committee members Dr. Sarika Khushalani Solanki and Dr. Muhammad Choudhry, whose feedback and reviews helped me improve the quality of this thesis.

I would like to thank my parents and my siblings who have supported me throughout entire process, both by keeping me harmonious and helping me putting pieces together. I will be grateful forever for your love.

Finally, I am extremely grateful for the emotional support selflessly given by all of my friends during my time here. There are quite probably too many to list that have contributed in ways large and small to this completed work. 


\section{TABLE OF CONTENTS}

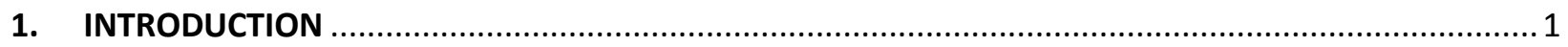

1.1. The Need for State Estimation in Power Systems ................................................................... 1

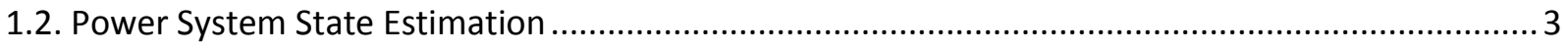

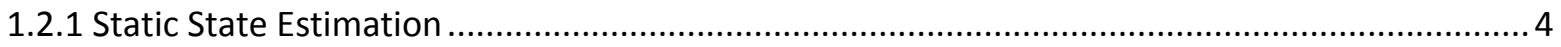

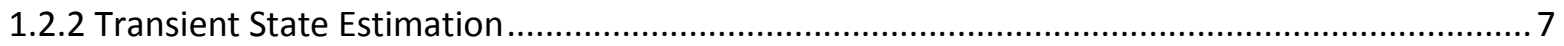

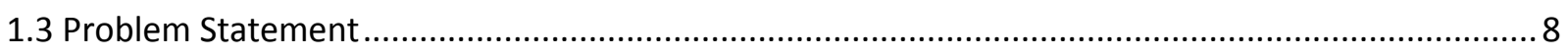

1.4 Thesis Outline

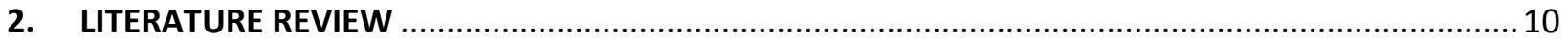

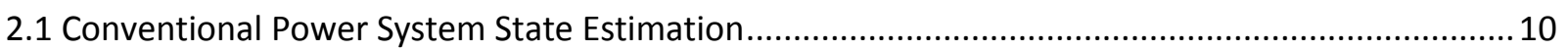

2.2 Distributed State Estimation Techniques ............................................................................ 13

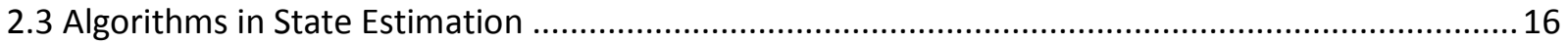

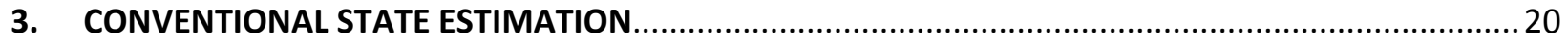

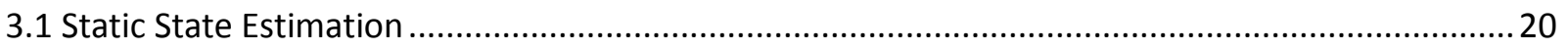

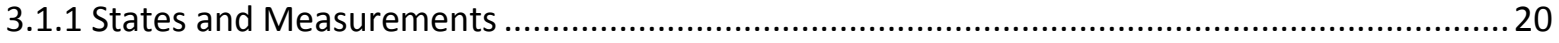

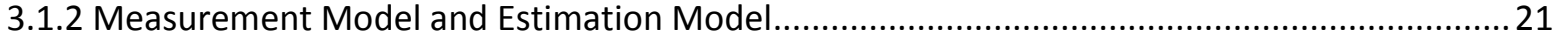

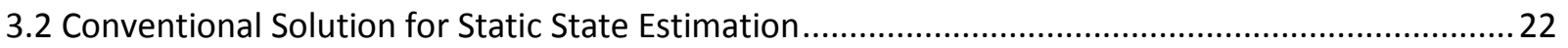

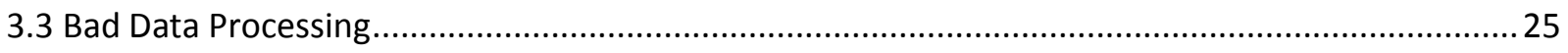

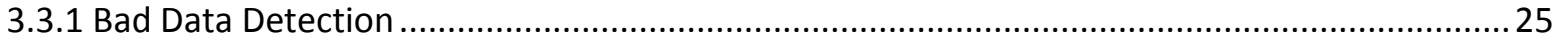

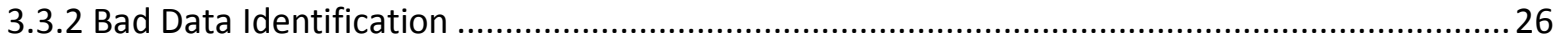

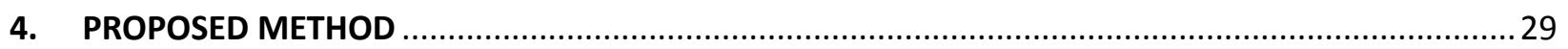

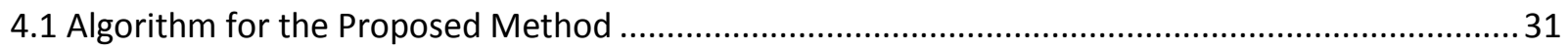

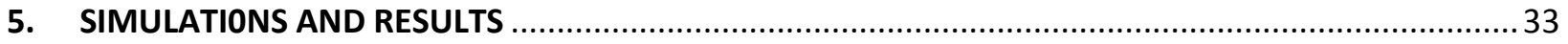

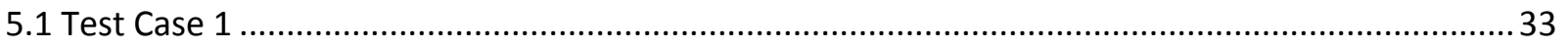

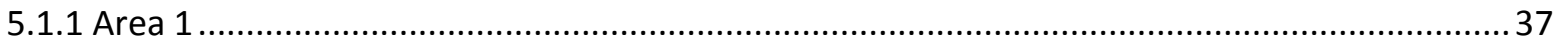

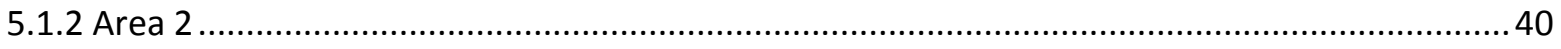

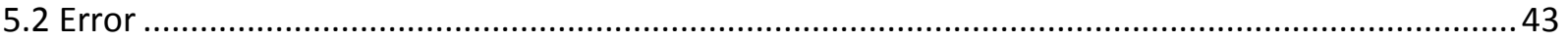

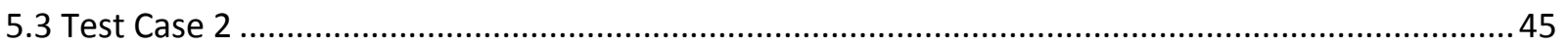

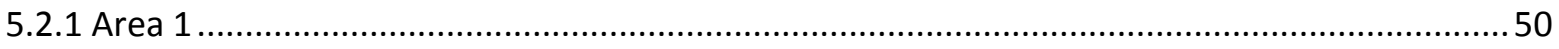


5.2.2 Area 2

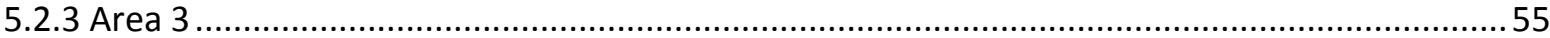

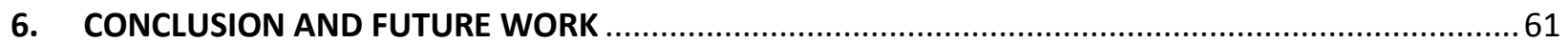

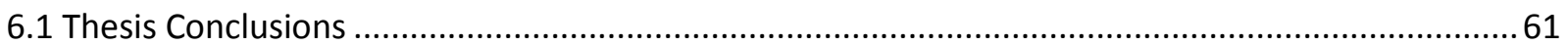

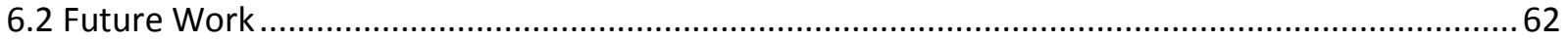

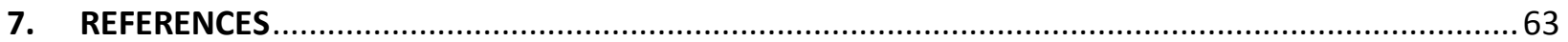




\section{LIST OF FIGURES}

Fig 2.1: Function diagram of practical state estimation .................................................................. 10

Fig 2.2: Communication scheme in hierarchical configuration ......................................................... 17

Fig 2.3: Communication scheme in decentralized configuration......................................................... 18

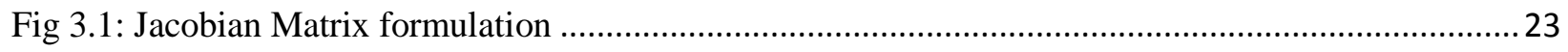

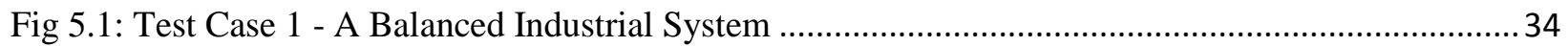

Fig 5.2: (a) Voltage magnitudes of 13-bus system, (b) Voltage angles of 13-bus system.........................36

Fig 5.3: (a) Voltage magnitude errors of 13-bus system, (b) Voltage angle errors of 13-bus system. .......36

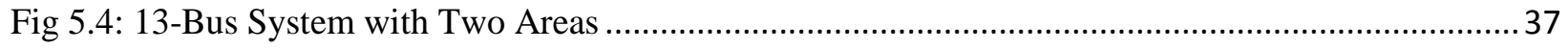

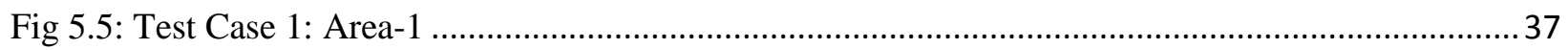

Fig 5.6: (a) Comparison of Voltage magnitudes of Area-1 with the centralized solution, (b) Comparison of Voltage angles of Area-1 with the centralized solution..................................................................... 39

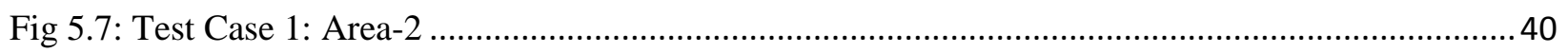

Fig 5.8: (a) Comparison of Voltage magnitudes of Area-2 with the centralized solution, (b) Comparison of Voltage angles of Area-2 with the centralized solution................................................................ 41

Fig 5.9: (a) Comparison of Voltage magnitudes of proposed method with the centralized solution, (b)

Comparison of Voltage angles of proposed method with the centralized solution.................................... 43

Fig 5.10: (a) Comparison of Voltage magnitudes of proposed method with the centralized solution, (b) Comparison of Voltage angles of proposed method with the centralized solution....................................45

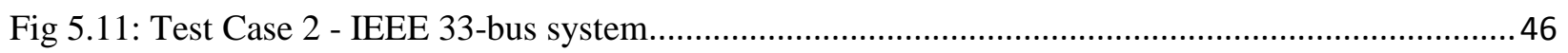

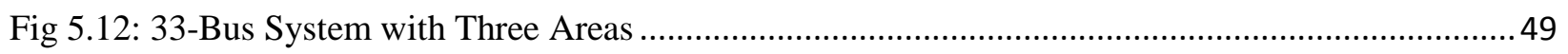

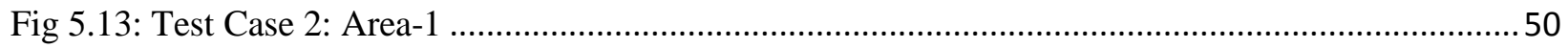

Fig 5.14: (a) Comparison of Voltage magnitudes of Area-1 with the measured values, (b) Comparison of Voltage angles of Area-1 with the measured values..........................................................................52

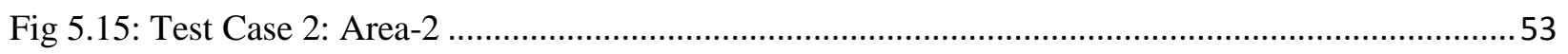

Fig 5.16: (a) Comparison of Voltage magnitudes of Area-2 with the measured values, (b) Comparison of Voltage angles of Area-2 with the measured values..............................................................................5

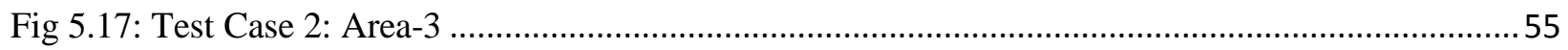

Fig 5.18: (a) Comparison of Voltage magnitudes of Area-3 with the measured values, (b) Comparison of

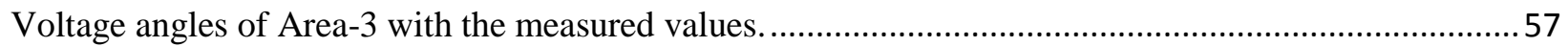

Fig 5.19: (a) Comparison of Voltage magnitudes of proposed solution with the estimated values, (b) Comparison of Voltage angles of proposed solution with the estimated values......................................60 


\section{LIST OF TABLES}

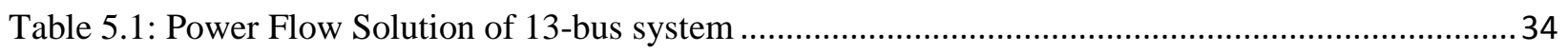

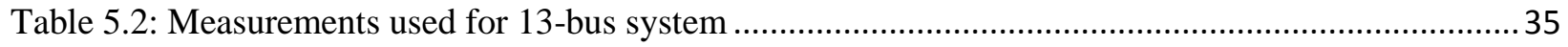

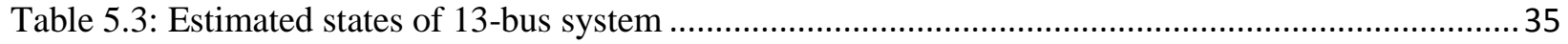

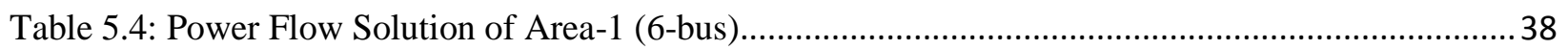

Table 5.5: Estimated states of Area-1 before the communication .............................................................39

Table 5.6: Power Flow Solution of Area-2 (8-bus) ................................................................................ 41

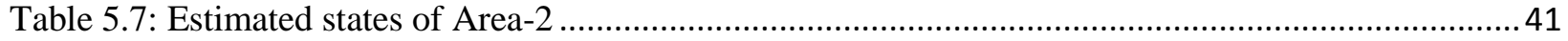

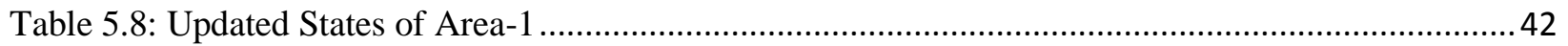

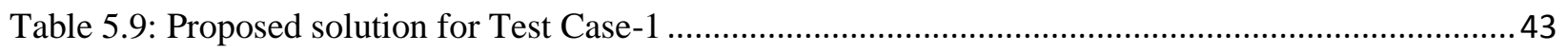

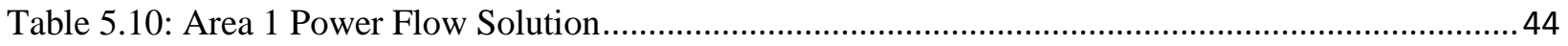

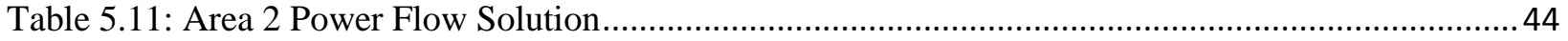

Table 5.12:Proposed solution with communication error ................................................................... 45

Table 5.13: Power Flow Solution of 33-bus system ....................................................................... 47

Table 5.14: Measurements used for 33-bus system .......................................................................... 48

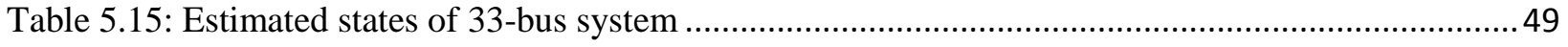

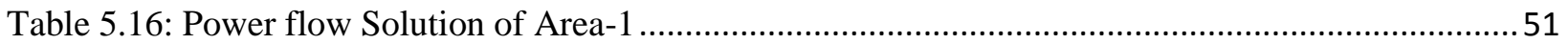

Table 5.17: Estimated states of Area-1 before the communication .....................................................52

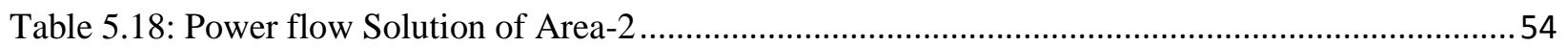

Table 5.19: Estimated states of Area-2 before the communication ......................................................54

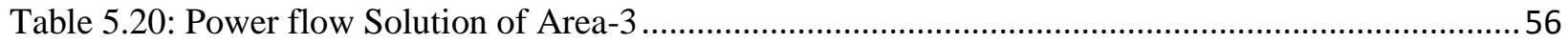

Table 5.21: Estimated states of Area-3 before the communication. ......................................................57

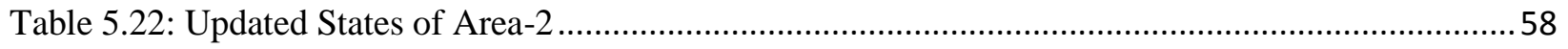

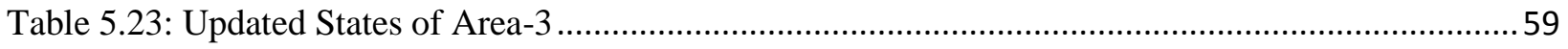

Table 5.24 : Proposed Solution for Test Case-2 …...........................................................................60 


\section{INTRODUCTION}

Electric power systems account for a critical part of our society's energy infrastructure. Over the years we have grown to depend on the near perfect reliability of these systems that have become a necessary part of our everyday lives. All of our household appliances, communication devices, and almost all of our tools ranging from construction sites to our offices require electricity for operation. It is not as if we assume electricity will always be available, it is that we believe electricity will always be available.

This kind of reliability doesn't happen without a great deal of effort from individuals such as electrical engineers and larger bodies such as electric utilities, universities, and government organizations. One of the key aspects to maintaining the reliability of a large system such as the electric power grid is finding a way to provide feedback to those that control it. Finding a way to accurately monitor the system has been the goal of engineers for the majority of the life of our electric grid. If system operators can be provided with appropriate information regarding the conditions of their systems, then they can use that information to make decisions that will improve not only the day-to-day reliability of the system but allow for engineers to plan more effectively for the future.

\subsection{The Need for State Estimation in Power Systems}

Outages like the one of Aug. 10th, 1996 and the one of Aug. 14th, 2003 have propelled the need for parameter estimation of synchronous generators and for situational awareness of the transmission system.

Following the 1996 blackout investigation, Western Systems Coordinating Council (WSCC) developed guidelines on synchronous machine model validation as a response to North American Electric Reliability Corporation (NERC) report on the outage [1]. Amongst the findings of the report, it was brought forward that machines parameters and states estimation play an important role in power system stability studies $[2,1]$. Nowadays transmission system is under stress as the generation and loading are constantly increasing and capacity of transmission lines has not 
increased proportionally. Therefore the transmission system must operate with ever decreasing margin from its maximum capacity. For this to happen operators need reliable information to operate. They need to have more confidence in the values of certain variables of interest than direct measurement can typically provide. Information delivery needs to be sufficiently robust so that it is available even if key measurements are missing. The interconnected power networks have become more complex. The task of securely operating the system has become more difficult.

In recent years, the electricity sector is facing several changes and challenges related to new legal and regulatory frameworks, to the explosion of dispersed generation and to larger pressures to increase quality of service. In distribution networks these challenges are perhaps even more evident clearly requiring larger investments on automation and telemetering devices as well as in the installation of more powerful control centers. This move determines the need to develop new methodologies and models to cope with specific characteristics of distribution networks.

Right from the development of the topic in the early 1970's [5], power system State Estimation (SE) has become a crucial part of the operation and management of transmission systems worldwide. Until recently, the application of SE at the distribution level, i.e. Distribution System State Estimation (DSSE) [31], has not been of significant interest. This is mainly because distribution networks have traditionally been designed and operated as passive systems, where power flows are unidirectional and relatively easy to predict and manage. However, distribution networks are seeing increasing penetrations of distributed energy resources, such as small to medium-sized Distributed Generation (DG), demand-responsive loads, electric vehicles and devices with storage capability. This has led to a requirement for improved observability in distribution systems, and the need for Distribution System Operators (DSOs) to take a more active role in monitoring and controlling the operation of the networks. DSSE has a crucial importance in this context.

The general problem addressed by the research described in this Thesis is the State Estimation problem in distribution networks. The State Estimation problem can be described as aiming at finding the values for a set of variables (state variables) that adjust in a more adequate way to a set of network values (measurements) that are available. The state variables are such that all the 
other network variables can be evaluated from them. The calculation of state variables considers the physical laws directing the operation of electrical networks and is typically done adopting some criteria.

This is not a complex problem if the number of network measurements is large, well distributed among the network and free of errors. However, in some networks the number of network measurements is reduced, there are some areas in the network where it does not exist any measurement and the available ones can be affected by errors or can even be incomplete. Therefore, for a system with these characteristics, the State Estimation turns into a challenge. In this thesis we will solve this problem by using all the information available for the network, not only measurement values. Of course, the quality of the solution turns better as the quality of the available information improves.

\subsection{Power System State Estimation}

Power system state estimation is "indeed a systematic procedure-a mathematical procedure-to process the set of real-time measurements to come up with the best estimate of the current state of the system" [4]. It utilizes redundant measurements from the system to compute the on-line states of buses in an estimator. The estimator is the hardware to perform state estimation. Normally, measurements include active and reactive power flows, active and reactive power injections and voltage magnitudes [4]. State estimation calculates the voltage magnitudes and phase angles of buses. These states (voltage magnitudes and phase angles) can be transmitted to and utilized in power system monitoring, controlling, dispatching, security analysis, etc.

The concept of state estimation was first proposed by Fred Schweppe in 1970 [5]. These publications are considered as the starting point of state estimation, and their importance has long been recognized in industry. Since Schweppe's proposals, state estimation has become an attractive topic, and a large amount of progress has been achieved to enhance its performance.

Typically, state estimation can be classified as static and transient state estimation according to the model employed. Static state estimation stands in a dominant position in the development of state estimation due to its reduced requirement of hardware. In this situation, the majority of 
researches are focused on static state estimation, whilst the publications about transient state estimation are rare. So far, all the practical estimators have employed static state estimation, and conventional state estimation, to some extent, can be considered as the static state estimation. Currently, state estimation is becoming the foundation of the EMS/SCADA system, and it is an indispensable part in control centers of power systems. The real-time results of state estimation can determine the accuracy of several functions in power system control and protection. In practical operation, the reliability is the most important factor in estimators, and several auxiliary procedures are integrated into estimators to guarantee the reliability. Several publications [4, 6] provide outlines of state estimation, and more comprehensive information can be referred to in $[3,8]$.

\subsubsection{Static State Estimation}

Compared with the transient one, static state estimation has lower hardware requirements, which makes its implementation simple in practical power systems. As a result, static state estimation attracted more attention in the development of state estimation. To some extent, conventional state estimation can be considered as the static state estimation.

Based on the load flow calculation and estimation theory, static state estimation was first proposed in 1970 [5]. It was defined as "a data processing algorithm for converting redundant meter readings and other available information into an estimate of the static-state vector", and as such it was used to deal with the uncertainties of measurements. In the practical operation, bad data was always appeared in the static state estimation, which had negative impacts such as the decrease in the estimation accuracy.

\section{a) Estimation process:}

Estimation process is the fundamental function in the static state estimation, and it determines states from redundancy measurements of the power system. A multitude of algorithms have been proposed to conduct this function. One successful method is the Weight Least Square (WLS) algorithm. 
- Development of the WLS algorithm

The WLS algorithm was proposed to solve the static state estimation, but its effect was not recognized by industry initially [5]. Fortunately, a revised version, based on the operational experience from power utilities, was developed soon and widely accepted. Since then, the research on the WLS algorithm has progressed greatly, and several aspects in the WLS estimation, including the sensitivity of measurements, the convergence quality of the estimation, the effects of weighting matrix, and the uncertainty of measurements have been investigated. These contributions promoted the development of the concept, model and solution of the WLS algorithm.

More methods have been proposed to improve the performance and provide greater applicability of the WLS algorithm. For instance, the fast decoupled load flow technique was introduced into the WLS estimation to reduce memory storage and improve computational efficiency [10]. A generalized state estimation considering the topology and parameter information was developed to provide greater applicability of the WLS algorithm, and a more robust algorithm for the generalized state estimation was proposed by applying mixed integer nonlinear program in the last decade. Recently, a modified WLS estimation utilizing historical measurements to calculate the auto tuning weights for new measurements was presented in [11], and higher estimation accuracy can be obtained.

- Alternative Formulations of the WLS algorithm

From the practical perspective, some inherent drawbacks in the WLS algorithm, such as unsatisfied convergence in a large system, limit its application. In addition, the WLS estimation is prone to be ill-conditioned, and the estimation is numerically unstable in this condition [3, 4]. To improve the robustness, researchers have proposed alternative formulations of the WLS algorithm. This improvement is mainly achieved through two methods: the Orthogonal Factorization and the Equality-Constrained.

In the WLS estimation, a crucial reason for the divergence problem is the intrinsically illconditioned gain matrix [3]. The Orthogonal Factorization methods were developed to avoid this ill-conditioned gain matrix by the factorization of the Jacobian matrix [12, 13]. The Golub's 
approach was first introduced to factorize the Jacobian matrix in [12]. This approach improved the numerical stability of the WLS algorithm, but at the same time, it brought a huge computational burden. In addition, the row ordering technique was introduced to improve efficiency [13]. In this attempt, the Jacobian matrix was reduced by rows, and the computational burden can be reduced effectively.

Another reason for the divergence problem is the use of virtual measurements in the WLS estimation, such as zero injections. The corresponding weights of these virtual measurements are very high, and this can tend to make the gain matrix ill-conditioned. On the other hand, these virtual measurements cannot be ignored, because the estimation accuracy may be decreased without them. The Equality-Constrained methods were proposed to model these virtual measurements as equality-constraints. Thus, these measurements can be excluded from the Jacobian matrix, and their large weights can be avoided. The Lagrangian multiplier was first introduced to solve the equality-constraints model in [14]. This method was effective, but it had an unsymmetrical matrix, which might lead to the computational difficulty. Subsequently, a matrix with the positive definite coefficient was employed to simplify the computation processes and improve the robustness. This method was further enhanced by the symbolic optimal ordering and the unique signed-Cholesky factorization in [15].

\section{b) Bad Data Processing:}

The bad data processing contains two processes: bad data detection and identification. The detection is to check the existence of bad data in the measurements of the state estimation. If the bad data is detected, the identification process starts to locate this bad data. The concept of the bad data processing was first defined in [9]. Three detection theories and two identification tests were proposed in [16] to deal with the bad data in the WLS estimation. One of them, the Largest Normalized Residual (LNR) test, can identify the single bad data easily and reliably, and thus it was widely accepted soon.

However, there are two drawbacks blocking the practical application of approaches in [16]. At first, the determination of the threshold in the detection process is difficult. A method, namely the Chi-square method, can be used to solve this problem [3]. This method is based on the fact that the objective function in the WLS estimation has a Chi-squares distribution. The other 
drawback is the lower accuracy of the bad data identification in case of multiple bad data. To increase the accuracy, the LNR test was revised to adjust multiple bad data.

The first attempt modified the LNR test by geometric integration. A novel algorithm was also established to introduce the measurement dependencies to the LNR test to recognize multiple bad data [17]. On the other hand, some scholars indicated that this modification of LNR test was inferior to satisfaction due to the inherent drawbacks of the test. In this situation, two improved methods, named as Estimation Identification (EI) and Hypothesis Testing Identification (HTI), were developed to replace the LNR test in bad data identification. In the EI approach, an inverse of the reduced residual sensitivity matrix was calculated and utilized to identify multiple bad data [18]. The HTI method estimated the errors of residuals to locate multiple bad data [19]. The HTI approach had less computational burden than the EI approach. Furthermore, several methods for multiple bad data identification in some special systems were proposed, such as in an unobservable system and in a non-uniquely observable system.

In addition, the alternative formulation of the WLS estimation can be also used to eliminate bad data. For instance, the state estimation problem was reformulated as a linear problem rather than a least square problem, and linear solution can be used to solve the problem correspondingly [20]. This approach reserved the degree of noise filtering and provided the capability of bad data rejection.

\subsubsection{Transient State Estimation}

The static state estimation is executed on the static model of the power system, and it was reasonable in the early stage due to hardware limitations. However, the dynamics of the power system could not be treated as static one in the practical situation. When some disturbances or faults happen, the power system experiences a transient process, and the static state estimation cannot satisfy the requirement of accuracy in this process. Hence, the transient state estimation is necessary, and some methods have been proposed to deal with the transient state estimation.

Initially, a discrete nonlinear observer was utilized to perform the transient state estimation. This method adopted the nonlinear differential equations to represent the transient model of the power system, and these equations were then discretized by the Taylor expansion. The above process 
became the foundation of the transient state estimation. Considering the synchronized machine, another attempt was published in [21]. An invariant imbedding non-linear dynamic method was used to estimate transient states in this attempt.

In the last decade, the progress of computer made the simulation of transient state estimation possible. Based on the state-space theory and the first-order differential equations, the Transient State Estimation (TSE) algorithm was proposed in [22]. Numerical simulations verified its effectiveness, but this algorithm was difficult to apply in practical power systems, because its hardware requirements cannot be satisfied. Another novel method was proposed to guide upgrading existed static state estimator to adjust the transient condition. This method was useful, but it cannot solve the problem fundamentally.

The potential use of PMU measurements in transient state estimation was discussed in the latest proposal [23]. This proposal recognized the capability of PMU measurements for capturing transients, and attempted to utilize these measurements, instead of solutions of conventional static state estimation, to form snapshots of power systems during the transient condition. However, the proposal focused on identifying transient incidents by PMU measurements rather than considering these measurements in the transient state estimation. Therefore, so far, no method can be used to perform the transient state estimation in practices.

\subsection{Problem Statement}

The major objective of this thesis is the proposal of a novel algorithm, namely the Consensus based Distributed State Estimation, utilizing decentralized configuration and conventional state estimation approach to mitigate the problem of low computational efficiency. The proposed algorithm is divided into two stages. In the first stage the balanced distribution system is divided into areas and using conventional WLS approach the states are estimated in respective areas. The next stage is to communicate between different areas which is done through agent based communication technique and then the states are updated depending on the messages received from the communication network. 
This thesis discusses the background information on the topic of distribution system state estimation and then presents the work done towards completing the goals through proposed algorithm. It begins with a brief history of the significance of power system state estimation followed by the presentation of the traditional form of state estimation. It continues with the development of the conventional state estimation equations and then investigates the planned implementation of a decentralized state estimation on two test systems and the associated research and software development. The developed algorithm is tested on 13 bus and 33 bus balanced distribution systems.

\subsection{Thesis Outline}

The remaining chapters of this thesis are organized as follows:

Chapter 2: The literature review on the historical development of state estimation and DSSE is presented. Also different algorithms of state estimation are presented.

Chapter 3: The formulation of proposed algorithm and its application in determining the states of a balanced distribution system is presented. The software used in implementing this algorithm is also discussed.

Chapter 4: This chapter presents different test systems considered, and simulation results of each stage of the proposed methodology are shown.

Chapter 5: In this final chapter the thesis is summarized and future research topics are discussed. 


\section{LITERATURE REVIEW}

\subsection{Conventional Power System State Estimation}

In general, state estimators check for errors in the system and network parameters and improve the system observability. Also state estimators provide an additional functionality of providing mitigation against measurement and communication system noise. A practical state estimator contains the following functions [8], and a simple function diagram is shown as Fig 2.1

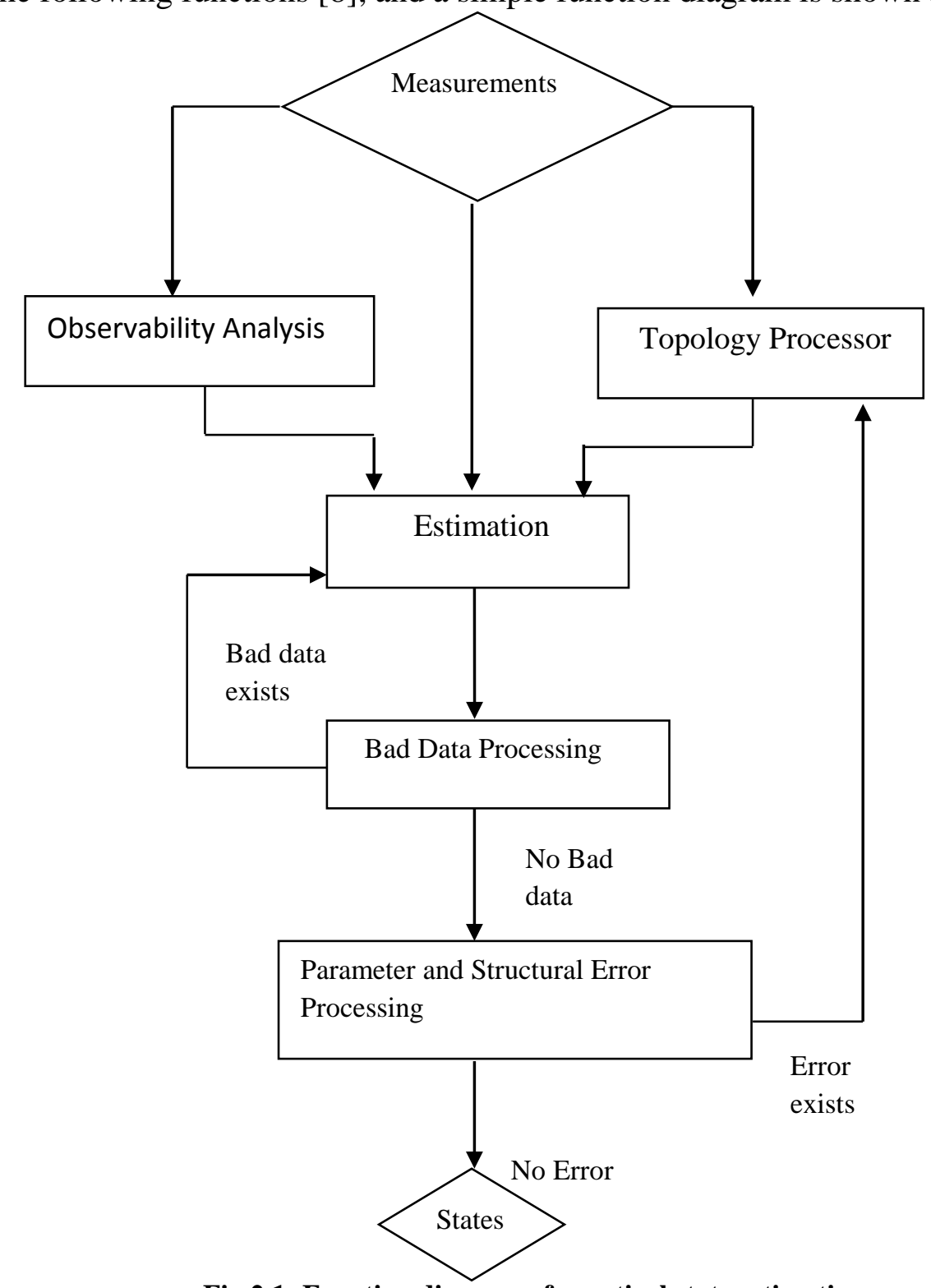

Fig 2.1: Function diagram of practical state estimation 
Topology Processor: Collects the status of circuit breakers and switches, and configures the topology of network.

$\checkmark$ Observability Analysis: Determines the observability of the system and recognizes unobservable islands if any exist.

$\checkmark$ Estimation: Estimates optimal states from redundant measurements and system model.

$\checkmark$ Bad Data Processing: Detects the existence of bad data in measurements and identifies bad data.

$\checkmark$ Parameter and Structural Error Processing: Estimates network parameters, detects structural errors in network configuration and locates errors if any exist.

\section{Formulation:}

The state is represented as the vector $\mathbf{x}$ (voltages and angles at each node) in the system. The measurements is given in the form of a vector, $\mathbf{z}$, to estimate $\mathbf{x}$. The measurements can be power/current injections or voltage magnitudes at system buses, active/reactive power flows in system branches, pseudo-measurements of network quantities, or any combination of the above. This forms a pre-determined set of non-linear equations[S],

$$
z=h(x)+e
$$

Where $h(x)$ - measurement functions corresponding to each measurement in $\mathrm{z}$

\section{e - Measurement error vector}

The objective function which $J(x)$ is to be minimized in this method is:

$$
\min _{x} J(x)=\min _{x}(z-h(x))^{T} \bar{W}(z-h(x))
$$

Where $\bar{W}$ : measurement weight matrix and the weights are set based on the inverse covariance of the respective metered measurements.

The minimization problem is solved iteratively [5], as:

$$
\Delta z_{n}=z-h\left(x_{n}\right)
$$




$$
\begin{array}{r}
\Delta x_{n}=\left(H^{T} \bar{W} H\right)^{-1} H^{T} \bar{W} \Delta z_{n} \\
\Delta x_{n+1}=x_{n}+\Delta x_{n}
\end{array}
$$

Where $H=\frac{\delta h(x)}{\delta x}$ is the Jacobian matrix and n: number of iterations.

Any bad data in the system is identified by applying statistical tests to both objective function $(J(\hat{x}))$ and to normalized residual vector $r=z-h(\hat{x})$. The normalization vector is given by $r_{n}=\rho_{j j}^{-1} r$, where $\rho_{j j}$ is the diagonal of the covariance matrix:

$$
C_{r}=\bar{W}^{-1}-H(\hat{x}) G^{-1} H^{T}(\hat{x})
$$

$J(\hat{x})$ Performance Index and Largest Normalized Residual Tests [8] are the most commonly used bad data statistical testing procedures.

There are some more state estimators in which the whole process remains the same except that the $J(x)$ will be replaced by different objective function. One example of this kind is:

\section{a) Weighted Least Average Value Estimator:}

The objective function [40] is given by

$$
\min _{x} J(x)=\bar{W}|z-h(x)|
$$

subject to $\left\{\begin{array}{l}f(x) \leq 0 \\ g(x)=0\end{array}\right.$

where $f(x)$ - vector of inequality constraints

$$
g(x) \text { - vector of equality constraints }
$$

In order to reduce the computational complexity, a fast coupled state estimator carrying out a direct current neglecting all branch resistances and shunt elements can be used. However, the assumptions and methods in this procedure are not valid for a distribution system and most methods cannot be implemented directly [32] on a distribution system. So, this paves way to look into a specifically designed distribution system state estimator. 


\subsection{Distributed State Estimation Techniques}

Power System State Estimation has always been of prime importance in the operation and management of transmission system. However, with the recent drive towards intelligent and more active power distribution networks, Distribution System State Estimation (DSSE) has been gaining significant research interest. The characteristics of distribution networks are different in many aspects with respect to transmission networks. Hence, many of the methods developed for "conventional" transmission level State Estimators cannot be directly applied to DSSE. This report provides an overview of the important techniques and algorithms available for DSSE with an additional application of Advanced Metering Infrastructure (AMI) data as inputs to the DSSE algorithms.

The fundamental differences between distribution and transmission systems are listed below:

- Construction: Transmission systems are generally meshed while the distribution systems have a radial network often with high $\mathrm{R} / \mathrm{X}$ ratios.

- Redundancy: Distribution systems are undetermined and have lower number of measurement points in comparison with transmission networks.

- Measurement Types: At the distribution level most of the available input data are measurements of power or current injections (pseudo measurements).

- Scale and Complexity: Due to the diversity of distribution systems and involvement of a large number of components the methods developed for DSSE need to be scalable, have a relatively low computational burden and be applicable for a range of different network types.

- Phase Imbalances: Distribution systems may have prominent phase imbalances which causes the need for a full three-phase system model unlike the conventional techniques.

In order to overcome these issues the following techniques have been developed:

- Adapting Conventional WLS Techniques to DSSE: Many methods focus on applying conventional WLS techniques to distribution systems [31], but this approach suffers from significant limitations especially when dealing with noisy input data and robustness. Also, the fast coupled methods and DC approximations generally applied in conventional 
SE do not work when applied to DSSE due to the radial construction of distribution systems and high $\mathrm{R} / \mathrm{X}$ ratios [34].

- Load Estimation for DSSE: Most often, DSSE depends on pseudo-measurements at each point in the load network which are based on historical data and load forecasts. These measurements have lower accuracy than the actual measurements. Also, in DSSE the number of telemetered devices providing system measurements is often limited resulting in bad data identification. The load estimation technique [35], for DSSE is one such technique to overcome the issues with respect to distribution systems.

- DSSE in Unbalanced networks: In [33], a branch-current-based SE methodology is developed in which the network branch currents are used to represent the system. Therefore, the Jacobian matrix $\mathrm{H}$ can be decoupled on a per-phase basis allowing conventional SE methods to be applied to distribution systems which are unbalanced, or have single-phase or two-phase lateral feeders.

\section{a) Forecast-Aided State estimation (FASE)}

In the SE systems discussed previously, the estimation is based only on the current state of input measurements and not on the earlier input data. In order to keep a track of the changes during normal operation specific SE techniques have been designed which recursively update the state estimate. FASE approaches uses following equations from [36] and the Extended Kalman Filter $(\mathrm{EKF})[37]$ :

$$
\begin{gathered}
x_{k+1}=F_{k} x_{k}+g_{k}+w_{k} \\
z_{k}=h_{k}\left(x_{k}\right)+v_{k}
\end{gathered}
$$

Where $F_{k}$-State Transition matrix

$g_{k}$-State trajectory behavioral vector

$w_{k}$ and $v_{k}$-process and observation noise corresponding to zero mean Gaussian noise.

The Jacobian matrix $H_{k}$ is calculated at every time step (k) with the current predicted states which are used in the EKF equations. An innovation analysis can be carried out in to determine if 
the new measurements are significantly different from the predicted values. This is done by making a short term forecast of the state variables each time a set of measurements becomes available. Therefore, this approach permits the detection of bad input data and network configuration or parameter errors because it filters the new input data using the Extended Kalman Filter (EKF) equations. In specific, if high resolution data is available from synchronized metering devices such as Phasor Measurement Units (PMU's) then FASE approaches are highly suitable for DSSE.

\section{b) Multi-area and Hierarchical DSSE Techniques}

One of the most challenging aspects of DSSE is the high level of computational complexity involved as it comprises of many thousands of individual nodes. All the measurements are typically processed in one centralized SE in a conventional system. However, in order to overcome the difficulty of handling a complex distribution system it is preferable to split the networks into a number of smaller sub-networks or "measurement areas". In this approach data is exchanged between areas only when they border each other and the SE is solved locally within each measurement area. The expression for multi-area SE is given as [38]:

$$
z_{m}=h_{m}\left(x_{m}\right), \quad \mathrm{m}=1 \ldots \mathrm{M} \text {. }
$$

Where $x_{m}=\left[x_{i m} x_{b m}\right]$, local measurement vector for area ' $\mathrm{m}$ ' containing the internal state variables $x_{i m}$, border state variables $x_{b m}$, for all the measurement areas, M.

The SE techniques have been developed separately for the transmission-level and the distribution-level. But the increasing requirement for communication and interaction between transmission and distribution network management systems has led to the development of multilevel or hierarchical SE's which integrate SE and DSSE.

c) Advanced Distribution Management Systems These systems are designed to optimize energy management in distribution networks [31]. Considering the need for better situational awareness and more active system support, this approach seems to be gaining importance even for distribution systems [39]. 


\subsection{Algorithms in State Estimation}

To achieve higher accuracy and efficiency, a multitude of algorithms have been proposed in state estimation. According to different configurations, these algorithms are usually divided into two groups, centralized algorithms and distributed algorithms. Generally, the distributed algorithms are developed from centralized ones to reduce computational burden. Some typical algorithms in these two groups are briefly introduced in this section.

\section{a) Centralized Algorithms}

In the centralized algorithms, the estimation for the overall system is performed at one time, and this can simplify the structure of these algorithms. The objectives of these centralized algorithms can be also divided into two categories.

The first category is to increase the applicability of the estimation. This is always achieved by considering the models of devices or special systems in the estimation.

For instance, some centralized algorithms were proposed to combine the model of the multiterminal system or FACTS devices in the estimation. The existing algorithms of transient state estimation [21, 22] are all belonged to this category, because the transient model of power systems is considered in them.

The other category is to improve the performance of the estimation. Two main options can achieve this target. Some algorithms are proposed to improve the estimation model, such as the optimization of solutions and factorization of matrices in the estimation. The alternative formulations of the WLS algorithm described above belong to this type. The other option is to introduce other mature techniques to the estimation. For example, the fast load flow technique was introduced in state estimation to improve computational efficiency [10].

b) Distributed Algorithms : The distributed algorithms are derived from the centralized algorithms to enhance computational efficiency [5]. The states in these algorithms are normally estimated in each subsystem individually, and this can reduce the computational burden in local estimations. In recent years, as a result of the rising of Smart Grids and the power industry reformation, the distributed configuration has attracted more attention 
than ever before [6]. It is certain that the distributed algorithms will be more significant in future state estimation. An early survey about these distributed algorithms was described in [24], and a more comprehensive introduction was presented in [7].

According to computational configuration, the structure of distributed algorithms could be divided into the hierarchical configuration and the decentralized configuration.

\section{- Hierarchical Configuration}

The hierarchical algorithms are constructed of the subsystem level and the coordination level. A large system is divided into a number of subsystems, and these subsystems constitute the subsystem level. The local estimation in each subsystem is performed separately. These local solutions are then coordinated at the upper level. Thus, the estimated results are only communicated between local estimators and the coordinator. This communication scheme is illustrated in Fig. 1.2.

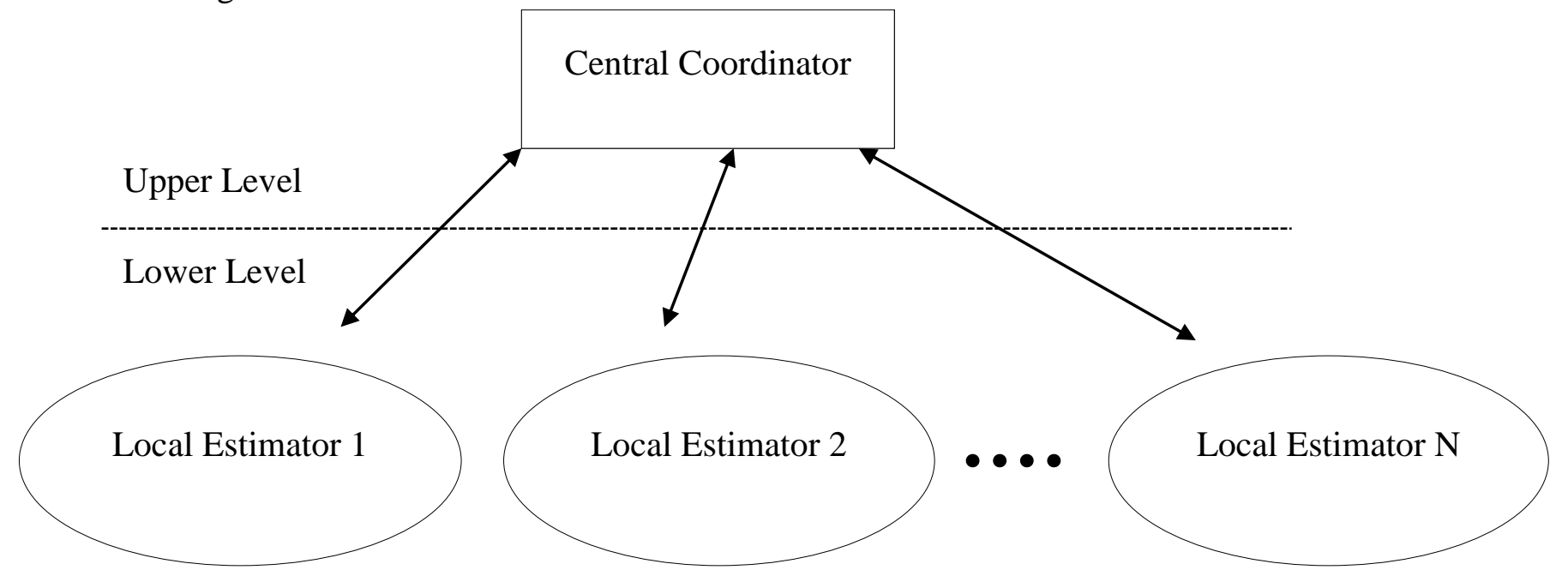

Fig 2.2: Communication scheme in hierarchical configuration

The hierarchical algorithms can be further divided into two groups by different coordination schemes. One group coordinates local results only once [25], and the other one conducts the coordination repeatedly [28].

The first group attracts more attention due to its simple implementation. In this group, the first method to re-estimate local results was published in [25], and this method utilized an overlapping strategy to decompose the large system. A novel algorithm was then proposed to 
replace the decomposition strategy as a non-overlapping one. The data process cost and local computer memory can be reduced in this method. A faster and more flexible algorithm was developed in [26] to further reduce computational burden and hence improve computational efficiency.

A reduced model with tie-line measurements was introduced to the hierarchical configuration to coordinate local results. Recently, [27] suggested a simple and efficient methodology to reduce the bandwidth requirements. This method only utilized the processed measurements other than raw measurements in the estimation.

- Decentralized configuration

As for the decentralized algorithms, the coordination process is not necessary. Local estimations are performed with the aids of boundary measurements from neighboring subsystems. The data is only communicated between adjacent subsystems, as shown in Fig. 1.3.

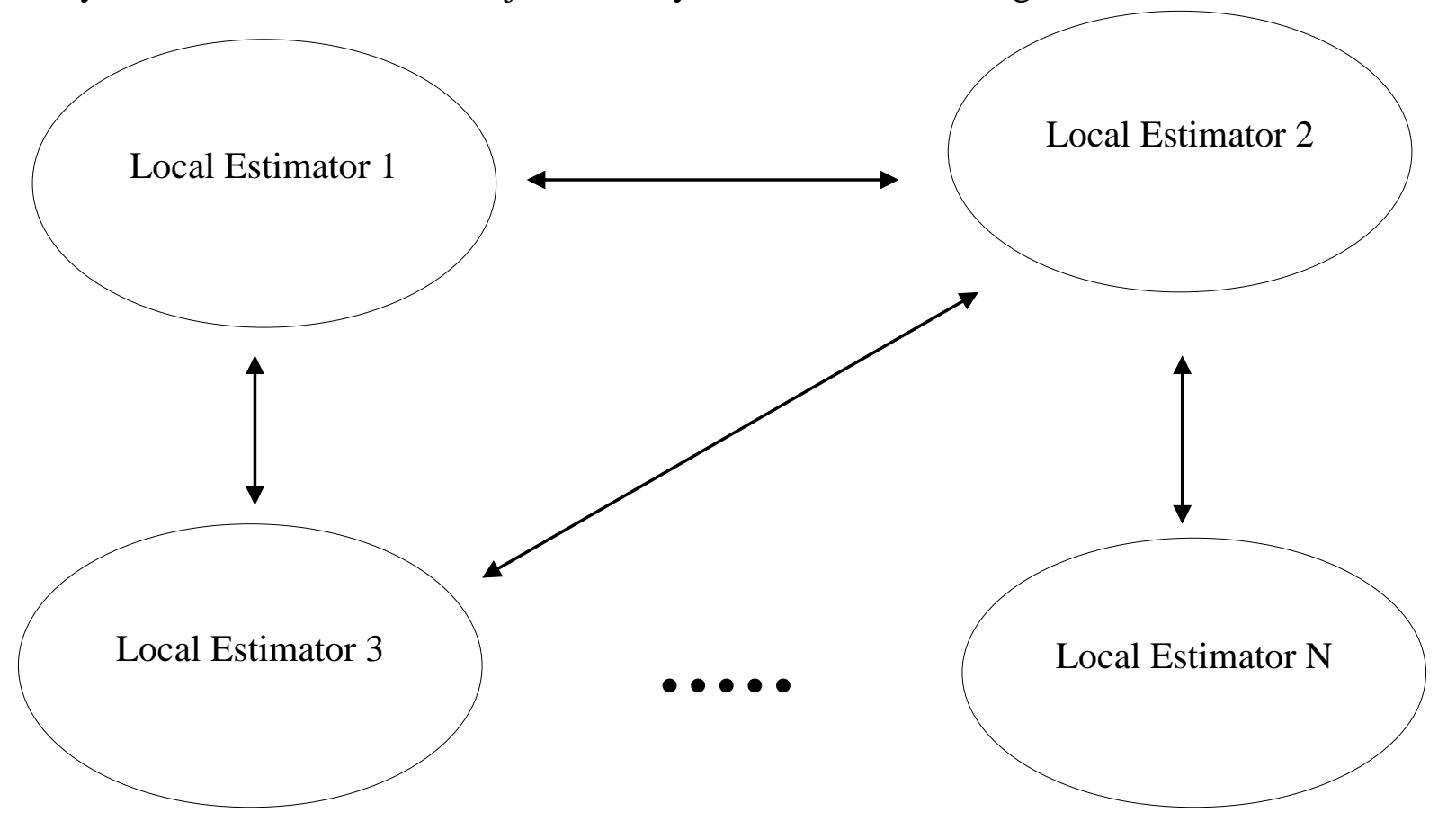

Fig 2.3: Communication scheme in decentralized configuration

Compared with the hierarchical algorithms, the decentralized algorithms require less on hardware, and their calculations are comparatively simple. So, the initial distributed state estimation algorithm employed this configuration [5]. Afterwards, some important progress 
promoted the development of decentralized algorithms [28]. The mature decentralized algorithm was proposed in [28]. The solutions from neighboring systems were assumed to be optimized, and this assumption was the theoretic basis to discard the coordination.

However, the convergence problem becomes worse in the decentralized algorithms, and the synchronization problem between local estimators is more serious. The convexity assumptions were introduced to the decentralized algorithms to deal with the convergence problem. A revised algorithm was proposed in [29], and it was more suitable for hardware implementation and onchip execution. In 2007, a novel decentralized procedure was proposed based on the optimization technique [30], and the robustness and applicability of this method were both improved. This thesis deals with decentralized estimation of voltage states for a radial distribution system. 


\section{CONVENTIONAL STATE ESTIMATION}

\subsection{Static State Estimation}

State estimation can be classified as static and transient state estimation according to different models (static model and dynamic model). The static model of power system requires less on calculation and hardware. On the contrary, it is still a challenge to estimate states with the dynamic model even now. Therefore, static state estimation usually attracted more attention in the development of state estimation. This chapter introduces the fundamental concept, model, and solution of the static state estimation, and presents a corresponding procedure of bad data processing.

\subsubsection{States and Measurements}

The objective of state estimation is to determine the states of buses from the redundancy measurements of the power system. Two essential elements in state estimation are measurements and states. In static state estimation, the state of each bus includes the voltage magnitude and the phase angle. This state can be defined as:

$$
x_{i}=\left[\begin{array}{ll}
v_{i} & \theta_{i}
\end{array}\right]^{T}
$$

Where

$x_{i} \quad$ State at Bus i;

$v_{i} \quad$ Voltage magnitude at Bus $\mathrm{i}$;

$\theta_{i} \quad$ Phase angle at Bus i;

All the measurements in conventional static state estimation are provided from RTUs. These RTU measurements are comprised of voltage magnitudes, active and reactive power flows, and active and reactive power injections, denoted by the subscript $v, p f, q f$, pinj and qinj respectively. Typically, RTU measurements $z$ can be defined as:

$$
z=\left[\begin{array}{lllll}
Z_{v} & z_{p f} & Z_{q f} & Z_{p i n j} & Z_{q i n j}
\end{array}\right]
$$




\subsubsection{Measurement Model and Estimation Model}

The measurement model in static state estimation demonstrates the relationship between the states and the measurements. Combined all these nonlinear relationships, the measurement model of the overall system can be obtained. This model and its compact form are expressed as [5]:

$$
\begin{gathered}
{\left[\begin{array}{c}
z_{1} \\
z_{2} \\
\vdots \\
z_{m}
\end{array}\right]=\left[\begin{array}{c}
h_{1}\left(x_{1}, x_{2}, \cdots, x_{n}\right) \\
h_{2}\left(x_{1}, x_{2}, \cdots, x_{n}\right) \\
\vdots \\
h_{m}\left(x_{1}, x_{2}, \cdots, x_{n}\right)
\end{array}\right]+\left[\begin{array}{c}
e_{1} \\
e_{2} \\
\vdots \\
e_{m}
\end{array}\right]} \\
z=h(x)+e
\end{gathered}
$$

Where

$z_{i} \quad$ Measurement $i$;

$h_{i}($.$) \quad Nonlinear function relating measurement i$ to states;

$e_{i} \quad$ Error of measurement $i$;

$\mathrm{z} \quad m \times l$ vector of measurements;

$\mathrm{x} \quad n \times 1$ vector of states;

The covariance matrix of measurement errors, denoted by $R$, is introduced to solve the measurement model above. The configuration of this covariance matrix is shown in (3.5), and it is formed on the corresponding standard deviation of independent measurements. It should be noticed that the measurement errors $e_{i}$ is Gaussian noise, i.e. $E\left(e_{i}\right)=0$, and $e_{i} \sim N(0, R i i)$, where $R i i$ is the $i^{t h}$ diagonal entry in the covariance matrix $R$.

$$
R=\operatorname{cov}(e)=\left[\begin{array}{llll}
\sigma_{1}^{2} & & & \\
& \sigma_{2}^{2} & & \\
& & \ddots & \\
& & & \sigma_{m}^{2}
\end{array}\right]
$$

Where

R $\quad m \times m$ covariance matrix of measurement errors, and it is a diagonal matrix;

$\sigma_{i} \quad$ Standard deviation of measurement $i$;

Afterwards, the estimation model of static state estimation is discussed. The estimation model is developed from the measurement model, and it represents the relationship between the 
measurements and the estimated results. Similar to the measurement model in (3.3) and (3.4), the estimation model and its compact form are formulated by:

$$
\begin{gathered}
{\left[\begin{array}{c}
z_{1} \\
z_{2} \\
\vdots \\
z_{m}
\end{array}\right]=\left[\begin{array}{c}
h_{1}\left(\widehat{x_{1}}, \widehat{x_{2}}, \cdots, \widehat{x_{n}}\right) \\
h_{2}\left(\widehat{x_{1}}, \widehat{x_{2}}, \cdots, \widehat{x_{n}}\right) \\
\vdots \\
h_{m}\left(\widehat{x_{1}}, \widehat{x_{2}}, \cdots, \widehat{x_{n}}\right)
\end{array}\right]+\left[\begin{array}{c}
r_{1} \\
r_{2} \\
\vdots \\
r_{m}
\end{array}\right]} \\
z=h(\hat{x})+r
\end{gathered}
$$

Where

$\widehat{x}_{l} \quad$ Estimated states at Bus $i$;

$r_{i} \quad$ Residual of measurement $i$;

$\hat{x} \quad n \times 1$ vector of estimated states;

$\mathrm{r} \quad m \times 1$ vector of residuals $(m>n)$.

The residuals of static state estimation demonstrate the differences between the estimated measurements and the exact ones. As for each measurement, the magnitude of the residual $r_{i}$ indicates the deviation extent between the estimated value and the actual value of measurement $i$. The sum of all these magnitudes or the absolute values of these residuals is used to show the progress of state estimation. When this sum reaches its minimum value, the estimation is finished, and the estimated states in (3.7) at the last iteration are the final estimated results.

\subsection{Conventional Solution for Static State Estimation}

There are several approaches that can be used to obtain the minimum sum of residuals. The Weight Least Square (WLS) algorithm is a popular one due to its greater applicability. The WLS algorithm aims to minimize the sum of the square weighted residuals. This aim can lead to an objective function $J(x)$, which is formulated as [3]:

$$
J(x)=(z-h(x))^{T} \cdot R^{-1} \cdot(z-h(x))
$$


When the above objective function reaches its minimum value, the estimated results of the WLS algorithm, denoted by $\hat{x}$, are obtained. Substituted (3.7) into (3.8), the minimum value of the objective function is expressed as:

$$
J(\hat{x})=(z-h(\hat{x}))^{T} \cdot R^{-1} \cdot(z-h(\hat{x}))=r^{T} \cdot R^{-1} \cdot r=\sum_{i=1}^{m} r_{i}^{2} R_{i i}
$$

To solve this minimized problem, the first order differential of $J(x)$ is introduced in (3.10), and it is denoted as $g(x)$ [3]. When the minimum value of $\mathrm{J}(\mathrm{x})$ is obtained, $g(x)$ equals 0 .

$$
g(x)=\frac{\partial J(x)}{\partial x}=-H^{T}(x) \cdot R^{-1} \cdot(z-h(x))
$$

In (3.10), $H(x)$ is called the Jacobian matrix, which is the first order differential of the nonlinear function in (3.4), i.e. $H(x)=\frac{\partial h(x)}{\partial x}$. The Jacobian matrix is very meaningful in the WLS algorithm and the corresponding bad data processing. Fig. 3.1 illustrates the configuration of the Jacobian matrix.

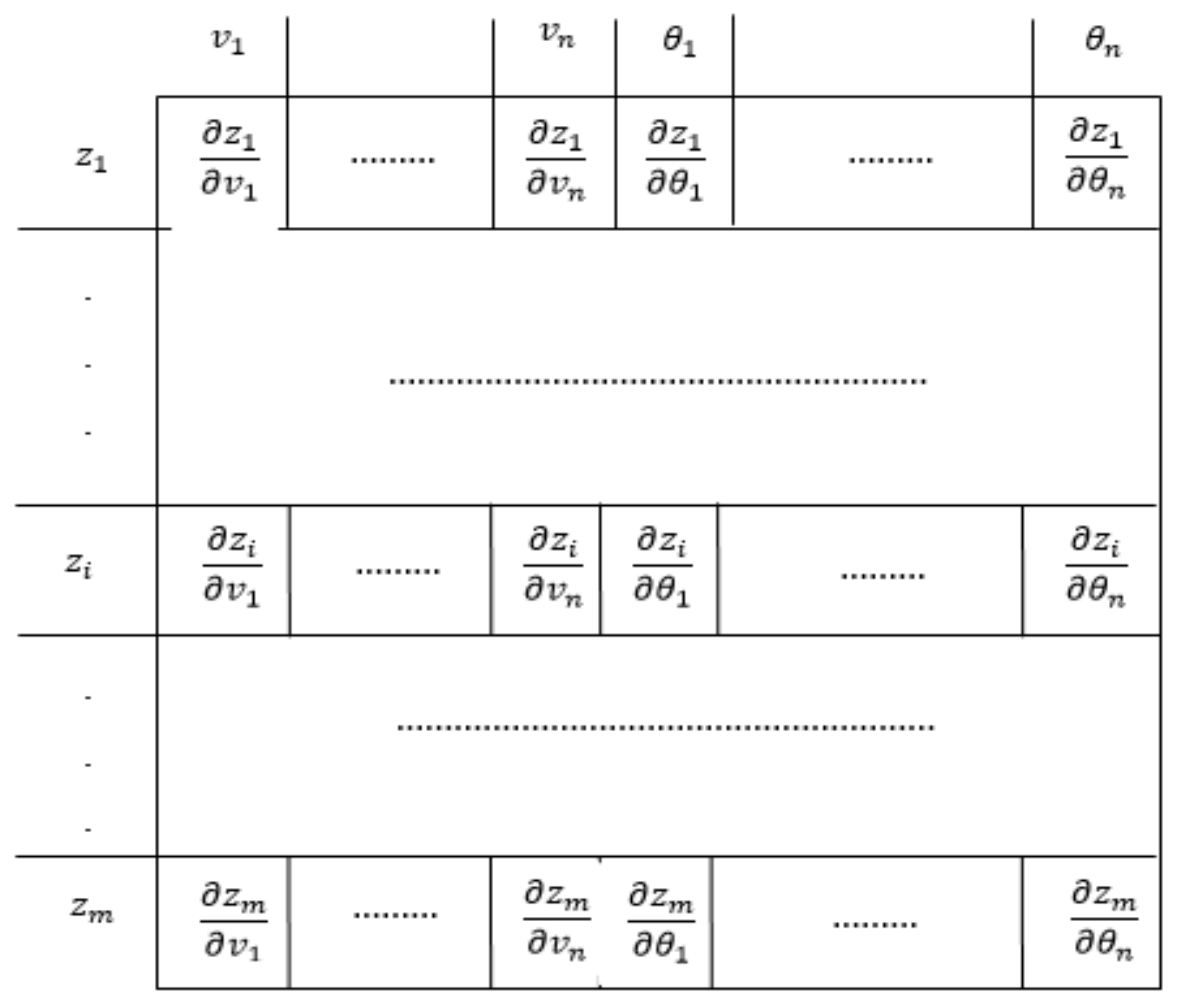

Fig 3.1: Jacobian Matrix formulation 
Thereafter, the (3.10) can be reformulated in (3.11) if the Taylor series of $g(x)$ is expanded at the vector $x^{k}$ and higher order components are ignored [3].

$$
g(x)=g\left(x^{k}\right)+\frac{\partial g\left(x^{k}\right)}{\partial x} \cdot\left(x-x^{k}\right)=g\left(x^{k}\right)+G\left(x^{k}\right) \cdot\left(x-x^{k}\right)=0
$$

Where $G(x)=\frac{\partial g}{\partial x} \quad$ - gain matrix

In (3.11), substitute $x$ with $x^{k+1}$, an iterative equation can be obtained, as shown in (3.12) [3]. The gain matrix $G(x)$ is introduced to denote the first order differential of $g(x)$. At the vector $x^{k}$, this gain matrix is formulated in (3.13).

$$
\begin{gathered}
g\left(x^{k+1}\right)=g\left(x^{k}\right)+G\left(x^{k}\right) \cdot\left(x^{k+1}-x^{k}\right)=0 \\
\Rightarrow x^{k+1}-x^{k}=-G\left(x^{k}\right)^{-1} \cdot g\left(x^{k}\right) \\
G\left(x^{k}\right)=\frac{\partial g\left(x^{k}\right)}{\partial x}=H^{T}\left(x^{k}\right) \cdot R^{-1} \cdot H\left(x^{k}\right)
\end{gathered}
$$

Where

$\mathrm{k} \quad$ iteration index;

$x^{k} \quad$ Estimated states at $\mathrm{k}$;

Finally, an iterative solution of the WLS algorithm is obtained by (3.10), (3.12), and (3.13). This solution is expressed in (3.14), and it is called the Normal Equation. It can calculate the vector $\Delta x$ at each iteration of the WLS algorithm.

$$
G\left(x^{k}\right) \cdot \Delta x^{k+1}=H^{T}\left(x^{k}\right) \cdot R^{-1} \cdot\left(z-h\left(x^{k}\right)\right)
$$

Where

$\Delta x^{k+1}=x^{k+1}-x^{k}$

This iterative calculation process would stop when the maximum value in $\Delta x$ is smaller than the convergence limit, which is normally chosen as 1e-6. 


\subsection{Bad Data Processing}

Measurement errors have always existed in practical estimators due to the limited accuracy of meters and the loss in the telecommunication medium. Small measurement errors are treated as Gaussian noise and their impacts can be neglected. On the other hand, some extremely large measurement errors caused by the wrong connections of meters, the failures of telecommunication system, and the incorrect measurements, etc., can damage the estimation. The measurements with these large errors are regarded as the bad data in state estimation.

The bad data can be classified as single bad data and multiple bad data. The single bad data is common in practical condition, and its detection and identification are simple. Because this thesis is mainly focused on the estimation process, only the single bad data is focused, and the multiple bad data will be considered in future. If the bad data exists, the estimation accuracy would decrease and the estimated results may be unacceptable. The bad data should be filtered first to guarantee the accuracy of results, and this procedure is called the bad data processing. This procedure always contains the processes of bad data detection and identification.

Regarding the WLS algorithm, the bad data can be only detected after the finish of the estimation due to the iterative estimation process in the algorithm. All the estimated results are checked by a detection process to determine the existence of the bad data. If there is bad data in the estimation, an identification process is conducted to locate and eliminate this bad data.

\subsubsection{Bad Data Detection}

A successful and widely applied approach to detect bad data in the WLS algorithm is the Chisquares approach. This approach is easy to implement and has a low computational burden. The precondition of the Chi-squares approach is that the objective function of the WLS algorithm conforms to the Chi-squares distribution. This is demonstrated by the following process. The objective function of the WLS algorithm is rewritten and simplified at first, as shown in (3.15) and (3.16).

$$
\begin{gathered}
J(x)=\sum_{i=1}^{m} R_{i i}^{-1}(z-h(x))^{2} \\
J(x)=\sum_{i=1}^{m} R_{i i}^{-1} e_{i}^{2}=\sum_{i=1}^{m}\left(\frac{e_{i}}{\sqrt{R_{i i}}}\right)^{2}=\sum_{i=1}^{m}\left(e_{i}^{N}\right)^{2}
\end{gathered}
$$


Where

$e_{i} \quad$ Error of measurement $\mathrm{i}$;

$R_{i i} \quad i^{\text {th }}$ Diagonal entry in the covariance matrix of measurement errors;

$e_{i}^{N} \quad$ Normalized error of measurement $i$.

Because the measurement error $e_{i}$ is Gaussian noise with the variance of $R_{i i}$, the normalized measurement error $e_{i}^{N}$ in (3.16) conforms to the Standard Normal Distribution [3], i.e. $e_{i}^{N} \sim N(0,1)$. Hence, the objective function of the WLS algorithm is demonstrated to obey the Chi-squares distribution. The Chi-squares distribution has $m-n$ degrees of freedom, where $m$ is the total number of measurements and $n$ is the total number of states. This is the theoretical foundation of the Chi-square approach in bad data detection. The Chi-square approach is executed according to the following steps.

Step 1 - calculate the objective function by (3.9) with the estimated results of the WLS estimation, as $J(\hat{x})$;

Step 2 - obtain the detection threshold from the Chi-squares distribution table with the detection confidence probability $p$ and the degrees of freedom $m-n$. Denote this threshold as $\chi_{m-n, p}^{2}$.

Step 3 - compare $J(\hat{x})$ with $\chi_{m-n, p}^{2}$.

If $J(\hat{x}) \geq \chi_{m-n, p}^{2}$, there is bad data in the WLS estimation.

Otherwise, the measurements are free of bad data.

\subsubsection{Bad Data Identification}

The bad data identification is more challengeable than detection, because it requires a more complex calculation and analysis to locate the bad data. The Largest Normalized Residual (LNR) approach is a simple and reliable method to identify the single bad data. This approach utilizes the normalized residual of each measurement. At first, the residual $i$ is the difference between the actual value and the estimated value of measurement $i$, and it can be calculated as:

$$
r_{i}=z_{i}-h_{i}(\hat{x})
$$


Afterwards, the residual sensitivity matrix $S$ is introduced to present the relationship between the residuals and the measurement errors in the WLS algorithm. This relationship is presented as:

$$
r=S . e
$$

According to the property of the WLS algorithm, the residual sensitivity matrix can be formulated as follows [3], where $I$ is the identity matrix.

$$
S=I-H \cdot G^{-1} \cdot H^{T} \cdot R^{-1}
$$

Because each measurement error conforms to Gaussian distribution, $e_{i} \sim N\left(0, R_{i i}\right)$, the mean value and covariance of the residuals can be solved from (3.18) as [3]:

$$
\begin{gathered}
E(r)=E(S . e)=S . E(e)=0 \\
\operatorname{Cov}(r)=E\left(r . r^{T}\right)=S . R=\Omega
\end{gathered}
$$

Where

$\Omega \quad$ Covariance of matrix residuals

Therefore, the residuals in the WLS algorithm also obey the Gaussian distribution, i.e. $r \sim N$ $(0, \Omega)$. Compared with the diagonal covariance matrix of measurement errors $R$, the residual covariance matrix $\Omega$ is an off-diagonal matrix. This is because the measurements in the estimation are independent, whilst the residuals may be correlated. This residual covariance matrix is calculated from (3.19) and (3.21) as:

$$
\Omega=S \cdot R=R-H \cdot G^{-1} \cdot H^{T}
$$

The diagonal entries of $\Omega$ are used to compute the normalized values of residuals. For each measurement, the normalized value of residual is calculated by its absolute value and the corresponding diagonal entry in $\Omega$ as: [3]

$$
r_{i}^{N}=\frac{\left|r_{i}\right|}{\sqrt{\Omega_{i i}}}
$$


Where

$r_{i}^{N} \quad$ Normalized residual of measurement $i$;

$\Omega_{i i} \quad i^{\text {th }}$ Diagonal entry in the covariance matrix of residuals.

These normalized residuals can identify the bad data. Detailed process of the LNR approach is summarized as follows:

Step 1, calculate the residual for each measurement by (3.17), as $r_{i}$;

Step 2, form the residual covariance matrix, $\Omega$, by (3.22);

Step 3, compute the normalized residual for each measurement by (3.23), denoted by $r_{i}^{N}$;

Step 4, find the measurement $j$ with the largest normalized residual $r_{j}^{N}$.

Step 5, compare the $r_{j}^{N}$ with the selected identification threshold, $\varepsilon$.

If $r_{j}^{N}>\varepsilon$, the measurement $j$ is recognized as bad data.

If not, all the measurements are free of bad data, and the bad data detection process need to be repeated.

Once the bad data is identified, it would be filtered in the estimation. Then, the state estimation and the procedure of bad data processing are repeated until no bad data exists. 


\section{PROPOSED METHOD}

"Smart Grid" is the future of electric power systems. The traditional electric power distribution systems are reforming by adapting intelligent agents and new communication technologies. The new smart distribution grid consists of two-way communication between electrically coupled neighbors and the end users who also act as agents. Such a system will ensure secured and reliable electric power delivery. Decentralized State estimation is one of the most important element of the smart distribution grid. Decentralized estimation is done with the help of sensor nodes acting as agents, observes only a part of the physical system and makes a local estimate of the entire system state with help of communication with the neighboring nodes. However full connectivity of sensor nodes is necessary for obtaining redundant local estimates which are equal to the one estimated in centralized system. As a result such a system, also called as Multi-Agent system requires more sensor nodes, which increases the number of communication links and also complexity of the system. Hence to solve this problem, sensor specific distributed state space model is introduced in which each sensor estimates the states for only a part of the overall physical system [43]. Thus the complexity associated with decentralized observation model is minimized. This thesis deals with decentralized estimation of voltage states for a radial distribution system. A good Multi Agent State Estimation (MASE) (hierarchical or decentralized architecture) must fulfill the following basic requirements: a) high computational efficiency, b) accuracy should be similar to the integrated solution, c) highly robust to deal with topology

changes, d) bad data processing for buses located close to boundary buses, and e) low data exchange between areas.

Static state estimation can be employed for determining the states of the smart distribution grid as long as rate of updating measurement set is greater than the underlying system dynamics. One of the most prominent static state estimation method based on observation space is Weighted Least Square (WLS) technique [44].WLS obtains states of the system that minimize the squared residual error, weighted by noise variances.

Let us consider a system whose state vector is $\mathrm{x}$ having $\mathrm{L}$ elements. In general, nonlinear relationship exists between measurements and $\mathrm{x}$ which can be described as:

$$
\mathrm{z}=\mathrm{h}(\mathrm{x})+\mathrm{e}
$$


Where,

$\mathbf{z} \in \mathbb{R}^{M}$ is the measurement vector

e is a vector of independent and identically distributed Gaussian noise with zero mean and covariance $C$.

$\mathrm{h}: \mathbb{R}^{L} \longrightarrow \mathbb{R}^{M}$ is a vector of functions that non-linearly maps state elements to measurement set z. For M>>L, the equation (4.1)[43] becomes over determined system of nonlinear equations.

The objective function is given as:

$$
\mathrm{J}=\arg \min _{x}[z-h(x)]^{T} C^{-1}[z-h(x)]
$$

The iterative Gauss-Newton algorithm is usually used for obtaining a solution to such a nonconvex problem. For this algorithm, the nonlinear measurement model obtained in equation (4.1) is approximated up to first order of the corresponding Taylor series expansion. At $(k+1)^{t h}$ the states are updated as:

$$
x^{(k+1)}=x^{(k)}+\left[H(k)^{-1} C^{-1} H(k)\right]^{-1} H(k)^{-1} C^{-1}\left[z-h\left(x^{(k)}\right)\right]
$$

Where, $\mathrm{H}$ is the jacobian matrix such that,

$[H(k)]_{m, l}=\left.\frac{\partial h_{m}(x)}{\partial x}\right|_{x=x^{(k)}}$

$\mathrm{H}$ is full-column rank which assures observability for over determined systems.

WLS is one of the methods employed for global state estimation from decentralized smart distribution system. Decentralization of radial distribution system is on a geographical basis done by decomposing the entire system into overlapping subareas [45]. Let us consider a physical system consisting of $\mathrm{N}$ subareas. The global state vector $\mathrm{x}$ is made up of $\mathrm{N}$ overlapped local state vectors $x_{1}, x_{2} \ldots \ldots x_{N}$.

The measurement model for $i^{\text {th }}$ subarea is given as:

$$
z_{i}=h_{i}\left(x_{i}\right)+e_{i} ; \quad i=1,2 \ldots \mathrm{N}
$$


And the global state vector is related to inter area boundary measurements as,

$$
z_{\text {boundary }}=h_{\text {boundary }}(x)+e_{\text {boundary }}
$$

Where, $\mathbf{z}$ is the measurement vector for $i^{\text {th }}$ subarea

$x_{i}$ is the state vector having only local elements of $i^{\text {th }}$ subarea

$e_{i} \sim \mathcal{N}\left(0, C_{i}\right)$

Respective local estimates of each subarea which are obtained using equation (4.3) are then centrally coordinated to obtain complete system states which are constrained to boundary conditions imposed by equation (4.5). Decentralized measurement model is used in order to find the estimate of the state of the system under the assumption that at some subareas global state might be unobservable.

In this thesis global consensus algorithm is employed such that in each subarea, global states are updated asynchronously. First allow the local estimators to converge to the desired tolerance, and then apply the coupling constraint corrections without any further local estimation iterations. After that the states in each area are updated accordingly based on the algorithm discussed later in the chapter and finally the global consensus will be achieved between the estimators thus giving the converged solution. This is implemented asynchronously as the values of the state variables in neighbor areas do not influence the local estimation iterative process and can be incorporated any time after the convergence of the local processes.

\subsection{Algorithm for the Proposed Method}

Step 1: START

Step 2: Consider a balanced radial distribution system. Run power flow for the entire system.

Step 3: Now the system states are to be estimated. This is done in two steps:

i. $\quad$ First step is to detect and identify any bad data present in the given measurements using Bad Data Processing.

ii. Next step is to estimate the states using Conventional WLS Estimation Technique. 
Step 4: Divide the system into areas such that there is one fictitious bus $i$ (or boundary bus) which is common to all the areas. This bus is used as an "agent" to communicate between the neighbors.

Step 5: The load at the fictitious bus $i$ is the net power at this bus i.e. the net power from all the neighboring areas. Similarly this is done for each area respectively.

Step 6: Run power flow for each area separately.

Step 7: Repeat "Step 3" for each area respectively.

Step 8: Compare the voltage angle of the agent containing slack bus with the angles of remaining agents:

- If the angles are equal

Proceed to next step

- else

Difference in the angles is added to each bus voltage angle of that respective area.

Step 9: STOP

In this chapter we just discussed about the proposed algorithm and how it works where as in the next chapter we will implement this proposed algorithm on two modified IEEE test systems and interpret the results. 


\section{SIMULATIONS AND RESULTS}

Before the test system is fed into the estimator, it must first be broken down into individual areas. The division of these areas do not play an important role in the second level solution, so the formation of the areas is subject to only one stipulation, the individual areas must be observable. If an individual area is unobservable, the first level state estimator will be unable to converge for that area. This will lead to missing information for the second level estimator, again causing a non-converging error. The proposed algorithm is implemented on two test systems: modified IEEE 13-bus systems and IEEE 33-bus systems and the implementation is done in MATLAB environment.

\subsection{Test Case 1}

This test case consists of 13 buses and is representative of a medium-sized industrial plant. The system is extracted from a common system that is being used in many of the calculations and examples in the IEEE Color Book series [41]. The plant is fed from a utility supply at $69 \mathrm{kV}$ and the local plant distribution system operates at $13.8 \mathrm{kV}$. The system is shown in Fig 5.1[42]. Due to the balanced nature of this example, only positive sequence data is provided. Capacitance of the short overhead line and all cables are neglected. The plant power factor correction capacitors are rated at 6000 kvar. As is typically done, leakage and series resistance of the bank are neglected in this study. In this system bus 100:UTIL-69 is used as the slack bus. The power flow solution of this system is shown in Table 5.1. 


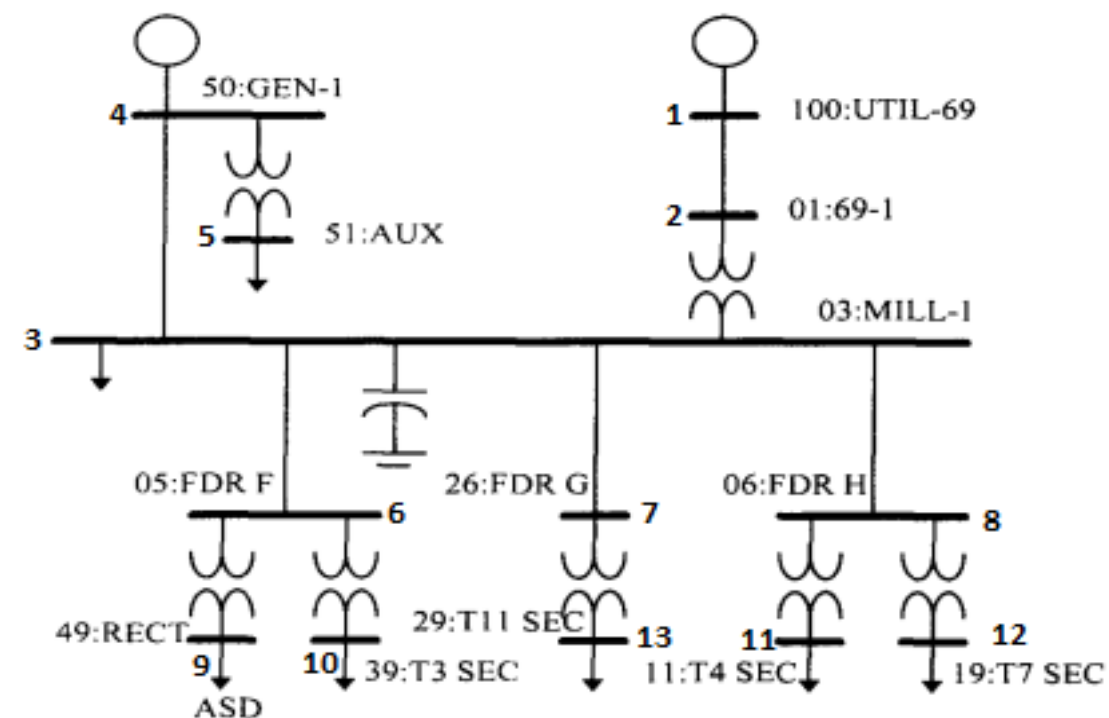

Fig 5.1: Test Case 1 - A Balanced Industrial System

\begin{tabular}{|c|c|c|c|c|c|c|}
\hline Bus No. & $\begin{array}{c}\text { Voltage } \\
\text { Mag.(p.u) }\end{array}$ & Angle(degree) & MW(Load) & Mvar(Load) & MW(Gen) & Mvar(Gen) \\
\hline 1. & 1.000 & 0.000 & 0.000 & 0.000 & 7.323 & 0.345 \\
\hline 2. & 0.999 & -0.122 & 0.000 & 0.000 & 0.000 & 0.000 \\
\hline 3. & 0.995 & -3.485 & 2.240 & -4.000 & 0.000 & 0.000 \\
\hline 4. & 0.995 & -3.474 & 0.000 & 0.000 & 2.000 & 1.783 \\
\hline 5. & 1.017 & -3.634 & 0.600 & 0.530 & 0.000 & 0.000 \\
\hline 6. & 0.994 & -3.488 & 0.000 & 0.000 & 0.000 & 0.000 \\
\hline 7. & 0.994 & -3.484 & 0.000 & 0.000 & 0.000 & 0.000 \\
\hline 8. & 0.994 & -3.484 & 0.000 & 0.000 & 0.000 & 0.000 \\
\hline 9. & 1.018 & -3.758 & 1.150 & 0.290 & 0.000 & 0.000 \\
\hline 10. & 1.039 & -3.854 & 1.310 & 1.130 & 0.000 & 0.000 \\
\hline 11. & 0.992 & -3.585 & 0.370 & 0.330 & 0.000 & 0.000 \\
\hline 12. & 1.032 & -4.238 & 2.800 & 2.500 & 0.000 & 0.000 \\
\hline 13. & 1.015 & -3.700 & 0.810 & 0.800 & 0.000 & 0.000 \\
\hline Total & & & 9.280 & 1.580 & 9.323 & 2.127 \\
\hline
\end{tabular}

Table 5.1: Power Flow Solution of 13-bus system 
Before dividing the system into areas the states of the entire system (centralized) are estimated using the Conventional WLS technique. This estimation is mainly done to compare the accuracy with the decentralized solution. Table 5.2 gives the details of measurements used and their respective covariance's. Table 5.3 is the solution to the centralized state estimation.

\begin{tabular}{|c|c|c|}
\hline Buses & Measurements & Measurement Error \\
\hline 1 & Voltage Magnitude & $64 \mathrm{e}-2$ \\
\hline 1,4 & Real Power Injection & $64 \mathrm{e}-2$ \\
\hline 1,4 & Reactive Power Injection & $64 \mathrm{e}-2$ \\
\hline $\begin{array}{c}1-2,2-3,4-3,4-5,3-6,3-7,3-8,6- \\
9,6-10,7-13,8-11,8-12\end{array}$ & Real Power Flows \\
$9,6-10,7-13,8-11,8-12$ & Reactive Power Flows & $64 \mathrm{e}-2$ \\
\hline
\end{tabular}

Table 5.2: Measurements used for 13-bus system

Meters- 12 for power flows and 2 for power injections

Number of measurements $: m=29$

Number of states to be estimated $: n=25$

\begin{tabular}{|c|c|c|}
\hline Bus No. & Voltage Mag.(p.u) & Angle(degree) \\
\hline 1. & 1.0004 & 0.0000 \\
\hline 2. & 0.9993 & -0.1209 \\
\hline 3. & 0.9936 & -3.4846 \\
\hline 4. & 0.9950 & -3.5015 \\
\hline 5. & 1.0285 & -3.7679 \\
\hline 6. & 0.9933 & -3.4873 \\
\hline 7. & 0.9933 & -3.4834 \\
\hline 8. & 0.9930 & -3.4830 \\
\hline 9. & 1.0170 & -3.7646 \\
\hline 10. & 1.0377 & -3.8740 \\
\hline 11. & 0.9908 & -3.5840 \\
\hline 12. & 1.0298 & -4.2789 \\
\hline 13. & 1.0139 & -3.7054 \\
\hline
\end{tabular}

Table 5.3: Estimated states of 13-bus system 


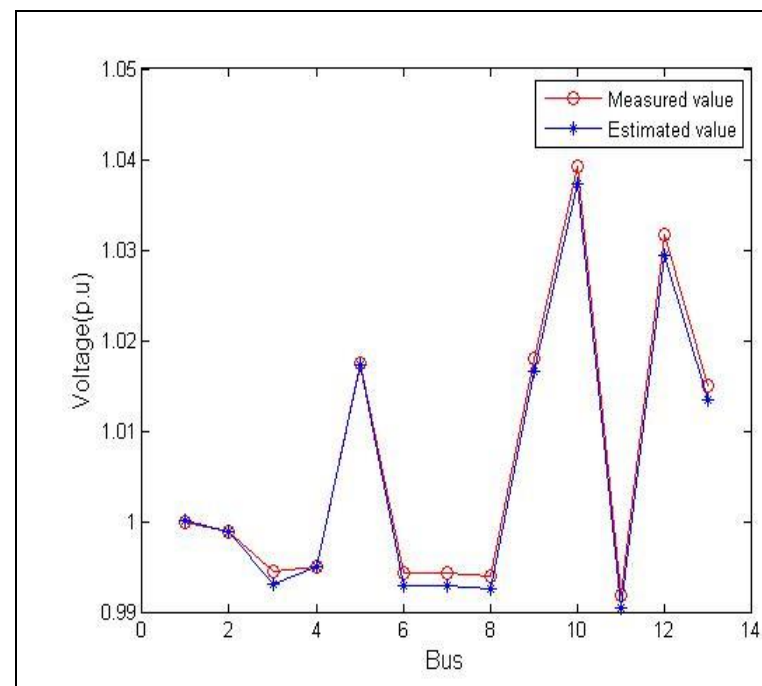

(a)

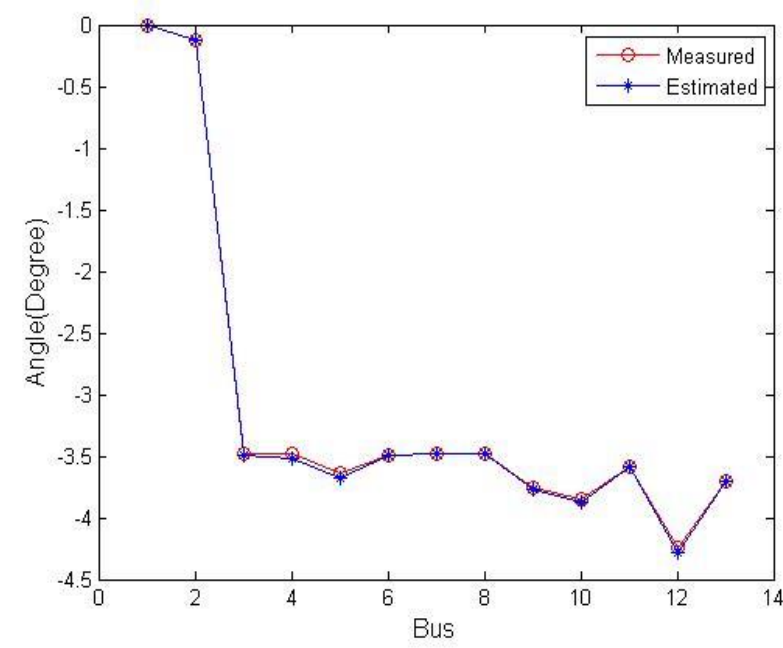

(b)

Fig 5.2: (a) Voltage magnitudes of 13-bus system, (b) Voltage angles of 13-bus system.

Fig.5.2 shows the comparison of the measured states with the estimated states which are obtained using WLS technique. As you can see that using this technique estimated values are almost equal to the measured values. Good accuracy can be obtained from this technique and this can be seen in Fig 5.3. Therefore from here on in this thesis wherever there is a need to estimate the states the conventional WLS technique will be used.

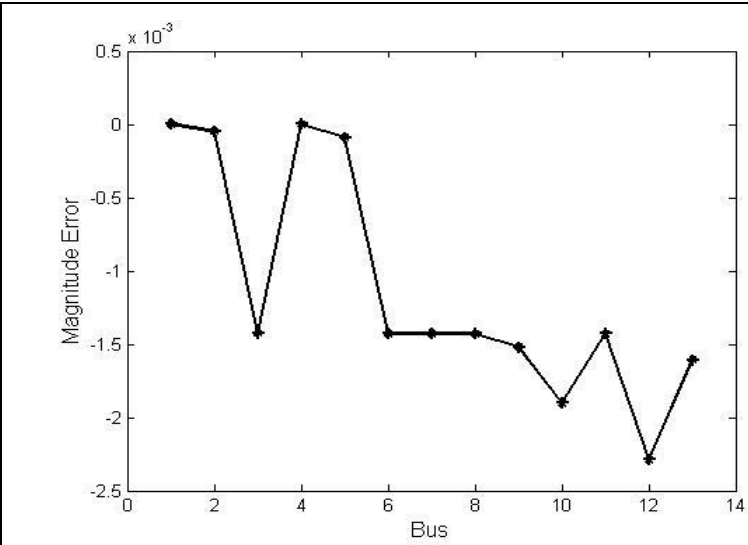

(a)

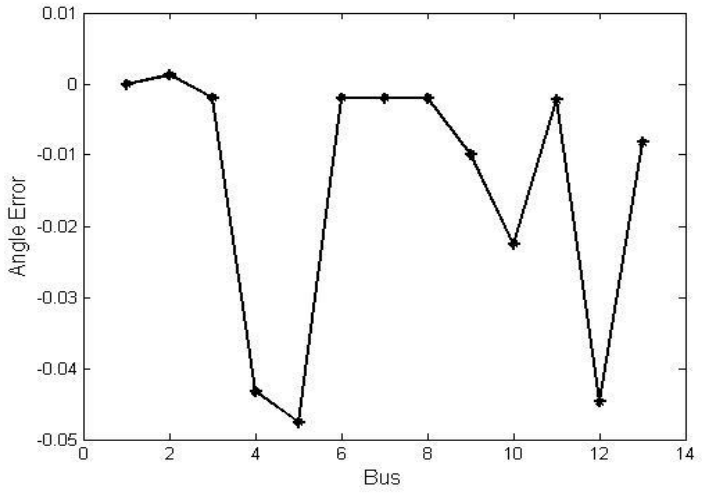

(b)

Fig 5.3: (a) Voltage magnitude errors of 13-bus system, (b) Voltage angle errors of 13-bus system.

Now the system is broken into two overlapping areas i.e. it has one bus (agent) common to both the areas. In this system Bus 3(MILL-1) is used as the agent. Fig.5.2 depicts the division of the 13-bus system into two overlapping areas. 


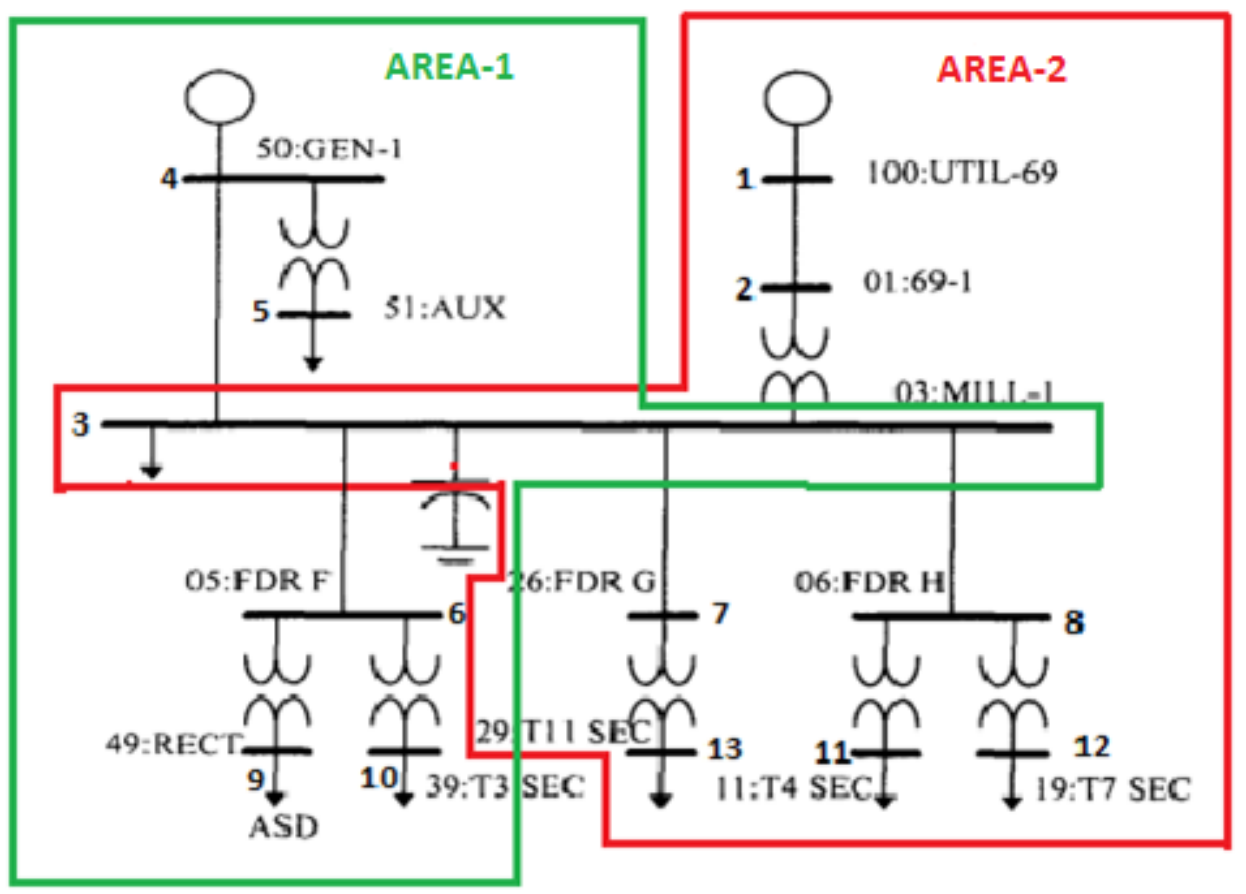

Fig 5.4: 13-Bus System with Two Areas

\subsubsection{Area 1}

This area consists of 6 buses out of which bus-1 i.e. 50 GEN-1 is used as the slack bus shown in green color in Fig.5.3. As said earlier bus-3 i.e. 03: MILL-1 is the agent.

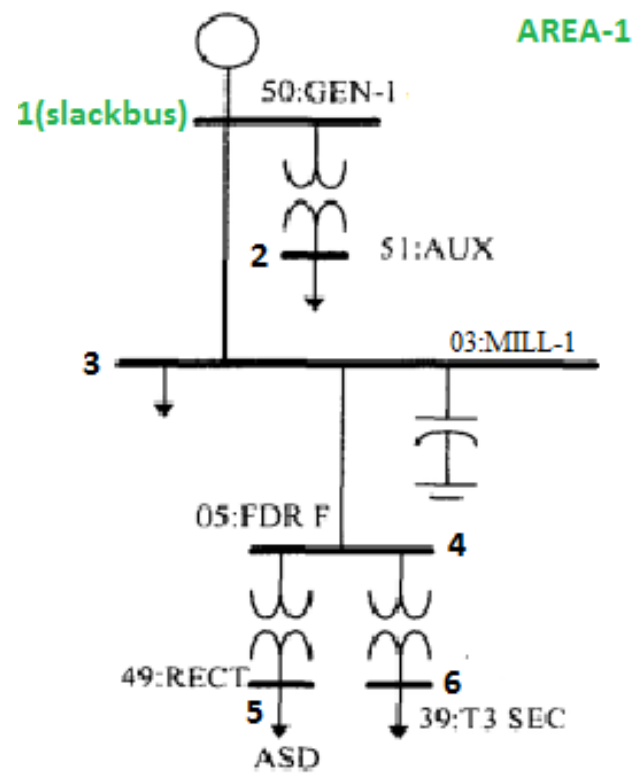

Fig 5.5: Test Case 1: Area-1 
Before performing power flow to this new system the agent's (bus-3) load is initialized using the formulas shown below:

$$
\begin{gathered}
\text { Real load } P_{L}(\text { bus }-3)=\sum_{i} P_{L(i)}-\sum_{k} P_{g(k)} \\
\text { Reactive load } Q_{L}(\text { bus }-3)=\sum_{i} Q_{L(i)}-\sum_{k} Q_{g(k)}
\end{gathered}
$$

Where

$i \quad$ - All the load buses in the neighboring areas i.e. Area-2 in this case.

$k \quad$ - All the generation buses in the neighboring areas i.e. Area-2 in this case.

$P_{L(i)}-$ Real load at $i_{t h}$ bus.

$Q_{L(i)}-$ Reactive load at $i_{t h}$ bus.

$P_{g(i)}-$ Real power generation at $k_{t h}$ bus.

$Q_{g(k)}$ - Reactive power generation at $k_{t h}$ bus.

Once the agent is initialized, power flow is run for this area. The power flow is done using Newton-Raphson method and the solution is shown in Table.5.4.

\begin{tabular}{|c|c|c|c|c|c|c|}
\hline Bus No. & $\begin{array}{c}\text { Voltage } \\
\text { Mag.(p.u) }\end{array}$ & Angle(degree) & MW(Load) & Mvar(Load) & MW(Gen) & Mvar(Gen) \\
\hline 1. & 0.995 & 0.000 & 0.000 & 0.000 & 1.956 & 1.255 \\
\hline 2. & 1.017 & -0.160 & 0.600 & 0.530 & 0.000 & 0.000 \\
\hline 3. & 0.995 & -0.014 & -1.103 & -0.715 & 0.000 & 0.000 \\
\hline 4. & 0.994 & -0.017 & 0.000 & 0.000 & 0.000 & 0.000 \\
\hline 5. & 1.018 & -0.286 & 1.150 & 0.290 & 0.000 & 0.000 \\
\hline 6. & 1.039 & -0.383 & 1.310 & 1.130 & 0.000 & 0.000 \\
\hline Total & & & 1.957 & 1.235 & 1.956 & 1.255 \\
\hline
\end{tabular}

Table 5.4: Power Flow Solution of Area-1 (6-bus) 
Now before communicating with the other area the states of this area are first estimated and using the WLS technique. The measurements and the measurement error values used here are same as the values used in Table.5.2. The estimated states are shown in Table 5.5.

\begin{tabular}{|c|c|c|}
\hline Bus No. & $\begin{array}{c}\text { Voltage } \\
\text { Mag.(p.u) }\end{array}$ & Angle(degree) \\
\hline 1. & 0.9950 & 0.000 \\
\hline 2. & 1.0173 & -0.1639 \\
\hline 3. & 0.9947 & -0.0140 \\
\hline 4. & 0.9944 & -0.0167 \\
\hline 5. & 1.0181 & -0.2934 \\
\hline 6. & 1.0389 & -0.4026 \\
\hline
\end{tabular}

Table 5.5: Estimated states of Area-1 before the communication

These estimated states are then compared with the states obtained from the centralized solution (Table 5.3) and it is assured that only the buses which are present in this area need to be compared. This is shown in Fig.5.6.

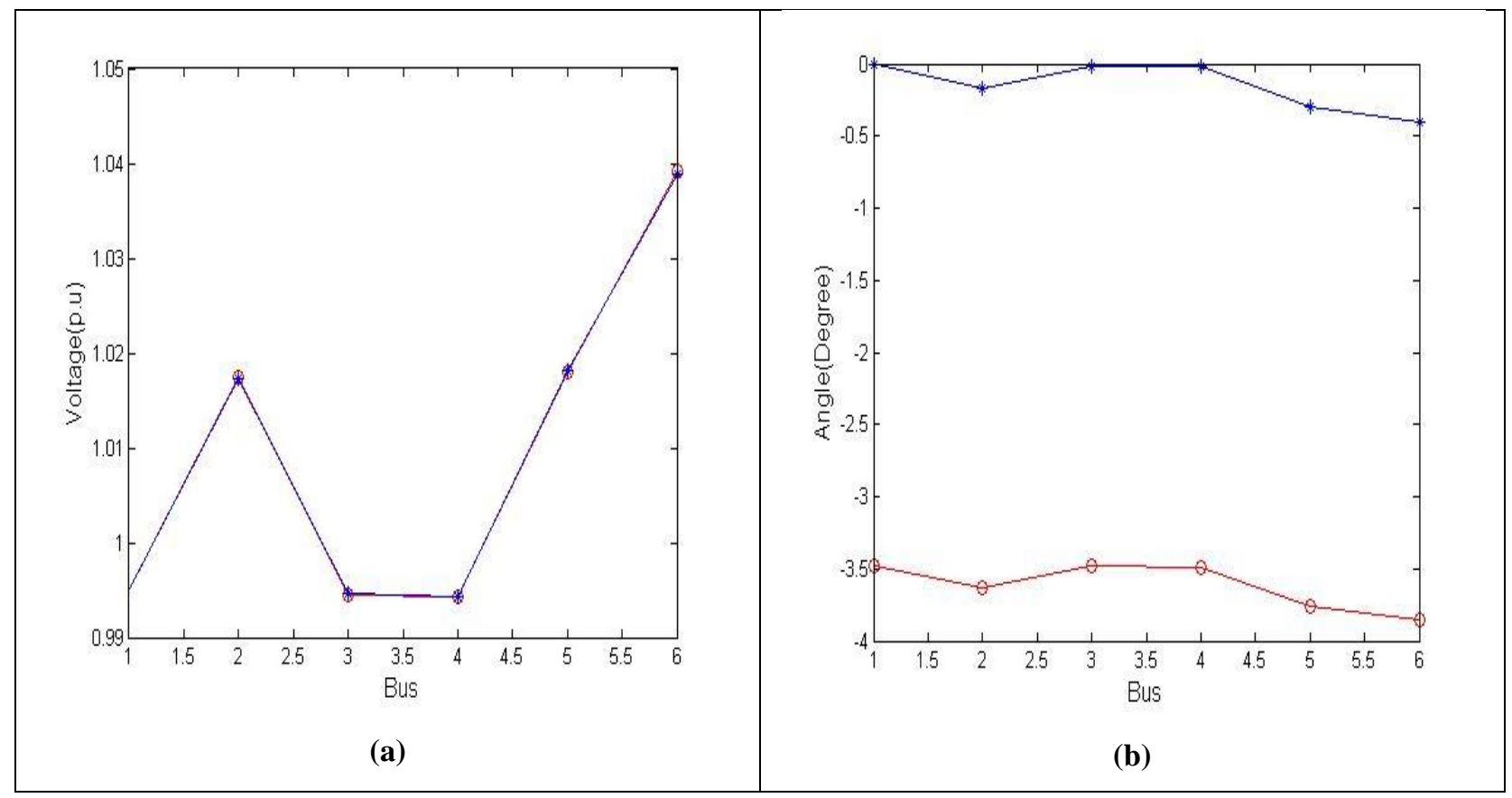

Fig 5.6: (a) Comparison of Voltage magnitudes of Area-1 with the centralized solution, (b) Comparison of Voltage angles of Area-1 with the centralized solution.

When we look at the above plots voltage magnitudes are converged but there is a big mismatch in the angles estimated and this can be minimized by communicating with the other areas. This whole point of this thesis is to achieve this communication strategy. 


\subsubsection{Area 2}

This area consists of 8 buses out of which bus-1 i.e. 100: UTIL-69 is used as the slack bus shown in red color in Fig.5.7. As said earlier bus-3 i.e. 03: MILL-1 is the agent.

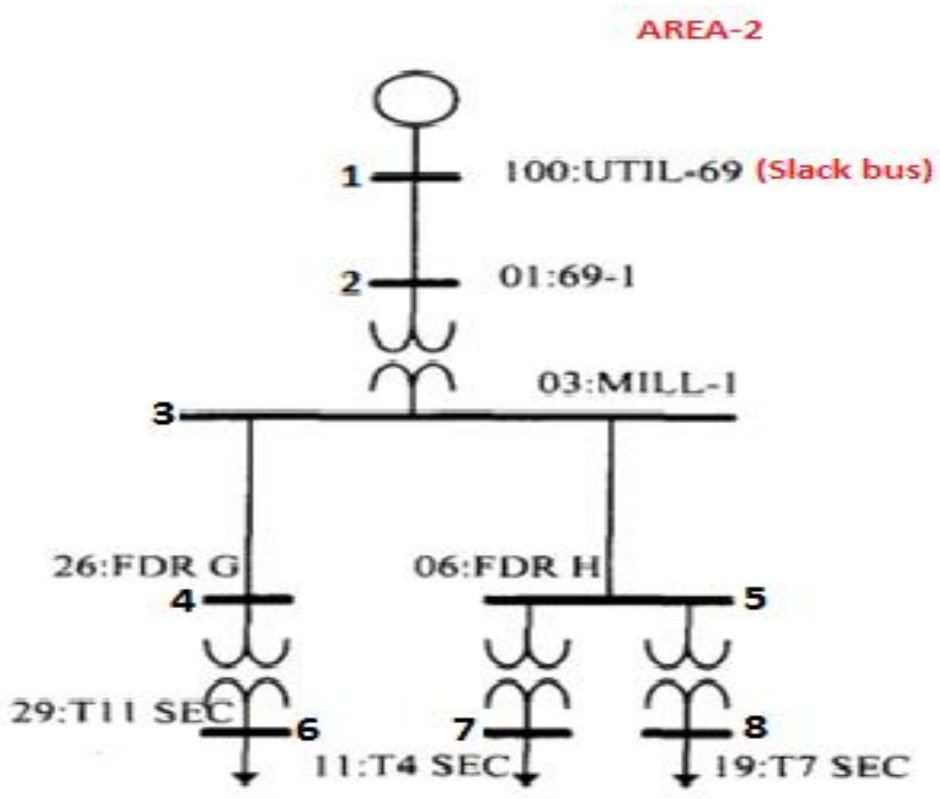

Fig 5.7: Test Case 1: Area-2

Again the agent is initialized (bus-3) before running the power flow and this is done by using the equations (5.1) and (5.2) where $i$ and $k$ are all the load buses and generation buses of Area-1 respectively. The power flow is done using Newton-Raphson method and the solution is shown in Table.5.6.

\begin{tabular}{|c|c|c|c|c|c|c|}
\hline Bus No. & $\begin{array}{c}\text { Voltage } \\
\text { Mag.(p.u) }\end{array}$ & Angle(degree) & MW(Load) & Mvar(Load) & MW(Gen) & Mvar(Gen) \\
\hline 1. & 1.000 & 0.000 & 0.000 & 0.000 & 7.315 & 0.323 \\
\hline 2. & 0.999 & -0.122 & 0.000 & 0.000 & 0.000 & 0.000 \\
\hline 3. & 0.995 & -3.482 & 3.300 & -3.833 & 0.000 & 0.000 \\
\hline 4. & 0.994 & -3.481 & 0.000 & 0.000 & 0.000 & 0.000 \\
\hline 5. & 0.994 & -3.481 & 0.000 & 0.000 & 0.000 & 0.000 \\
\hline 6. & 1.015 & -3.697 & 0.810 & 0.800 & 0.000 & 0.000 \\
\hline 7. & 0.992 & -3.581 & 0.370 & 0.330 & 0.000 & 0.000 \\
\hline
\end{tabular}




\begin{tabular}{|c|c|c|c|c|c|c|}
\hline 8. & 1.032 & -4.234 & 2.800 & 2.500 & 0.000 & 0.000 \\
\hline Total & & & 7.280 & -0.203 & 7.315 & 0.323 \\
\hline
\end{tabular}

Table 5.6: Power Flow Solution of Area-2 (8-bus)

Now before communicating with the other area the states of this area are first estimated using WLS technique. The measurements and the measurement error values used here are same as the values used in Table.5.2. The estimated states are shown in Table 5.7.

\begin{tabular}{|c|c|c|}
\hline Bus No. & $\begin{array}{c}\text { Voltage } \\
\text { Mag.(p.u) }\end{array}$ & Angle(degree) \\
\hline 1. & 1.0000 & 0.000 \\
\hline 2. & 0.9989 & -0.1217 \\
\hline 3. & 0.9947 & -3.4822 \\
\hline 4. & 0.9945 & -3.4810 \\
\hline 5. & 0.9941 & -3.4806 \\
\hline 6. & 1.0151 & -3.7024 \\
\hline 7. & 0.9920 & -3.5814 \\
\hline 8. & 1.0310 & -4.2743 \\
\hline
\end{tabular}

Table 5.7: Estimated states of Area-2

These estimated states are then compared with the states obtained from the centralized solution (Table 5.3) and it is assured that only the buses which are present in this area should be compared. This is shown in Fig.5.8.

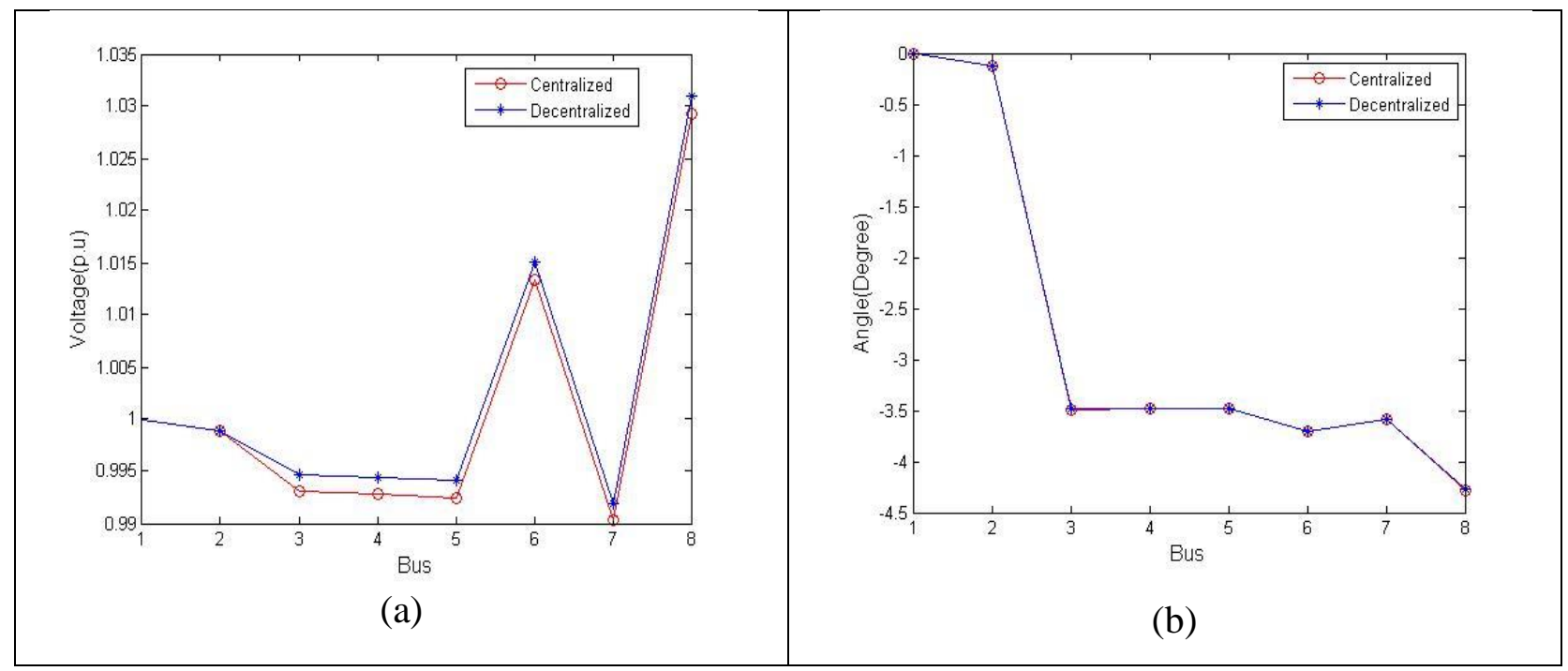

Fig 5.8: (a) Comparison of Voltage magnitudes of Area-2 with the centralized solution, (b) Comparison of Voltage angles of Area-2 with the centralized solution 
When we look at the above plots both the voltage magnitudes and the angles are converged unlike in the area-1 where the angles had a big mismatch. This is because area- 2 has the same slack bus as in original test system (13-bus). So this point will be used in developing a strategy to communicate between the two areas.

As we used bus-3(03: MILL-1) as an agent and it is common to both the areas so the states of this bus should be equal to the bus-3 used in the area-1. Using this criteria, communication is done between the two areas and the difference in the agent angles is calculated. This difference in angles is added to all the bus angles including the agent present in the neighboring area i.e. Area-1 in this case. If you compare the values of bus 3 angles from the two tables: Table 5.5 and Table 5.7 the difference is given by:

$$
\Delta \text { Angle }=-3.4822-(-0.0140)=-3.4682
$$

This value of $\Delta$ Angle is added to all the bus angles present in area-1 through which consensus is finally achieved. The new updated states in area-1 are shown in Table 5.8.

\begin{tabular}{|c|c|c|}
\hline Bus No. & $\begin{array}{c}\text { Voltage } \\
\text { Mag.(p.u) }\end{array}$ & Angle(degree) \\
\hline 1. & 0.9950 & -3.4682 \\
\hline 2. & 1.0173 & -3.6320 \\
\hline 3. & 0.9947 & -3.4822 \\
\hline 4. & 0.9944 & -3.4849 \\
\hline 5. & 1.0181 & -3.7616 \\
\hline 6. & 1.0389 & -3.8707 \\
\hline
\end{tabular}

Table 5.8: Updated States of Area-1

The two local estimators are then combined by assigning the states to each bus accordingly. We arrive at the final decentralized solution for this test case and this is shown in Table 5.9.

\begin{tabular}{|c|c|c|}
\hline Bus No. & $\begin{array}{c}\text { Voltage } \\
\text { Mag.(p.u) }\end{array}$ & Angle(degree) \\
\hline 1. & 1.0000 & 0.000 \\
\hline 2. & 0.9989 & -0.1217 \\
\hline 3. & 0.9947 & -3.4822 \\
\hline 4. & 0.9950 & -3.4682 \\
\hline
\end{tabular}




\begin{tabular}{|c|c|c|}
\hline 5. & 1.0173 & -3.6320 \\
\hline 6. & 0.9944 & -3.4849 \\
\hline 7. & 0.9945 & -3.4810 \\
\hline 8. & 0.9941 & -3.4806 \\
\hline 9. & 1.0181 & -3.7616 \\
\hline 10. & 1.0389 & -3.8707 \\
\hline 11. & 0.9920 & -3.5814 \\
\hline 12. & 1.0310 & -4.2743 \\
\hline 13. & 1.0151 & -3.7024 \\
\hline
\end{tabular}

Table 5.9: Proposed solution for Test Case-1

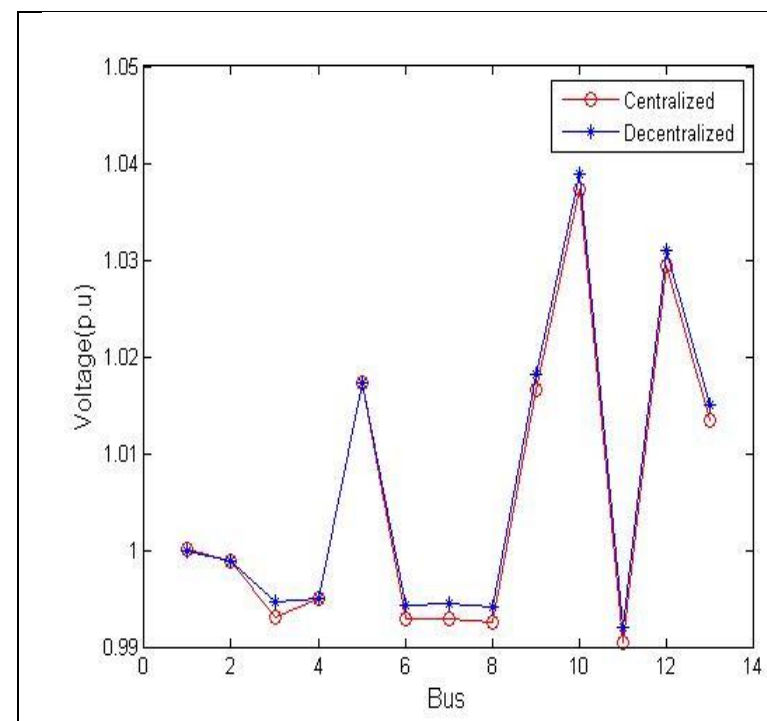

(a)

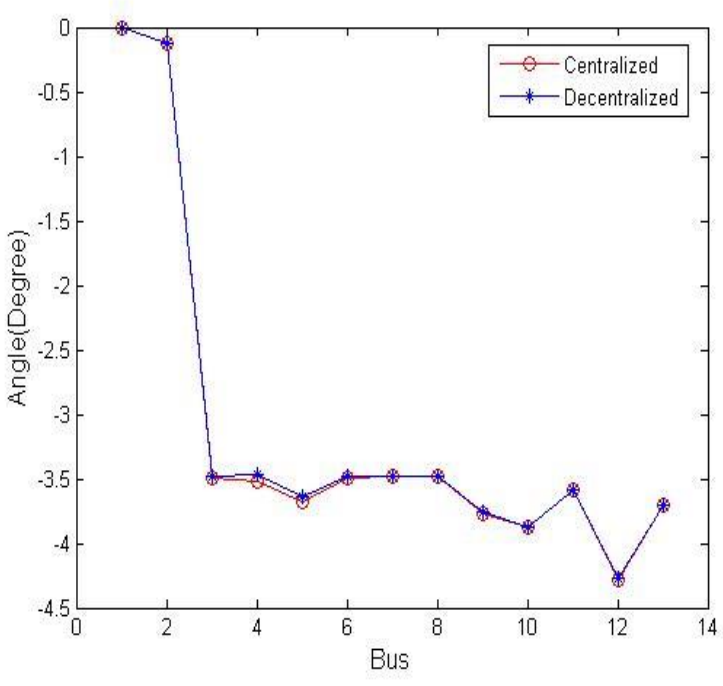

(b)

Fig 5.9: (a) Comparison of Voltage magnitudes of proposed method with the centralized solution, (b) Comparison of Voltage angles of proposed method with the centralized solution

\subsection{Error}

The previous case considered was ideal case but in practice the error should be included while communicating between the areas. The equations (5.1) and (5.2) are now represented as:

$$
\begin{array}{r}
\text { Real load } P_{L}(\text { bus }-3)=\left(\sum_{i} P_{L(i)}-\sum_{k} P_{g(k)}\right)+E R R O R \\
\text { Reactive load } Q_{L}(\text { bus }-3)=\left(\sum_{i} Q_{L(i)}-\sum_{k} Q_{g(k)}\right)+E R R O R
\end{array}
$$

In this thesis around 10 percent error is introduced i.e. adding Error $=0.3 \mathrm{Mw}+\mathrm{j} 0.3 \mathrm{Mvar}$ to the actual load at bus 3(Agent) in both the areas and the respective power flow solutions are shown in Table 5.10 and Table 5.11. 


\begin{tabular}{|c|c|c|c|c|c|c|}
\hline Bus No. & $\begin{array}{c}\text { Voltage } \\
\text { Mag.(p.u) }\end{array}$ & Angle(degree) & MW(Load) & Mvar(Load) & MW(Gen) & Mvar(Gen) \\
\hline 1. & 0.995 & 0.000 & 0.000 & 0.000 & 2.257 & 1.555 \\
\hline 2. & 1.017 & -0.160 & 0.600 & 0.530 & 0.000 & 0.000 \\
\hline 3. & 0.995 & -0.016 & -0.803 & -0.415 & 0.000 & 0.000 \\
\hline 4. & 0.994 & -0.019 & 0.000 & 0.000 & 0.000 & 0.000 \\
\hline 5. & 1.018 & -0.289 & 1.150 & 0.290 & 0.000 & 0.000 \\
\hline 6. & 1.039 & -0.385 & 1.310 & 1.130 & 0.000 & 0.000 \\
\hline Total & & & 2.257 & 1.535 & 2.257 & 1.555 \\
\hline
\end{tabular}

Table 5.10: Area 1 Power Flow Solution

\begin{tabular}{|c|c|c|c|c|c|c|}
\hline Bus No. & $\begin{array}{c}\text { Voltage } \\
\text { Mag.(p.u) }\end{array}$ & Angle(degree) & MW(Load) & Mvar(Load) & MW(Gen) & Mvar(Gen) \\
\hline 1. & 1.000 & 0.000 & 0.000 & 0.000 & 7.618 & 0.664 \\
\hline 2. & 0.999 & -0.124 & 0.000 & 0.000 & 0.000 & 0.000 \\
\hline 3. & 0.992 & -3.625 & 3.600 & -3.533 & 0.000 & 0.000 \\
\hline 4. & 0.992 & -3.624 & 0.000 & 0.000 & 0.000 & 0.000 \\
\hline 5. & 0.991 & -3.624 & 0.000 & 0.000 & 0.000 & 0.000 \\
\hline 6. & 1.012 & -3.841 & 0.810 & 0.800 & 0.000 & 0.000 \\
\hline 7. & 0.989 & -3.725 & 0.370 & 0.330 & 0.000 & 0.000 \\
\hline 8. & 1.029 & -4.382 & 2.800 & 2.500 & 0.000 & 0.000 \\
\hline Total & & & 7.580 & 0.097 & 7.618 & 0.664 \\
\hline
\end{tabular}

Table 5.11: Area 2 Power Flow Solution

Using this data the states of each area are estimated separately and the communication is done between the areas based on the same criteria discussed in earlier case. Finally combining both the areas, the proposed solution with the addition of error is shown in Table 5.12.

\begin{tabular}{|c|c|c|}
\hline Bus No. & $\begin{array}{c}\text { Voltage } \\
\text { Mag.(p.u) }\end{array}$ & Angle(degree) \\
\hline 1. & 1.0000 & 0.000 \\
\hline 2. & 0.9988 & -0.1229 \\
\hline 3. & 0.9946 & -3.4838 \\
\hline 4. & 0.9950 & -3.4692 \\
\hline
\end{tabular}




\begin{tabular}{|c|c|c|}
\hline 5. & 1.0167 & -3.6584 \\
\hline 6. & 0.9943 & -3.4866 \\
\hline 7. & 0.9944 & -3.4827 \\
\hline 8. & 0.9940 & -3.4823 \\
\hline 9. & 1.0181 & -3.7633 \\
\hline 10. & 1.0388 & -3.8724 \\
\hline 11. & 0.9919 & -3.5830 \\
\hline 12. & 1.0309 & -4.2761 \\
\hline 13. & 1.0150 & -3.7041 \\
\hline
\end{tabular}

Table 5.12:Proposed solution with communication error

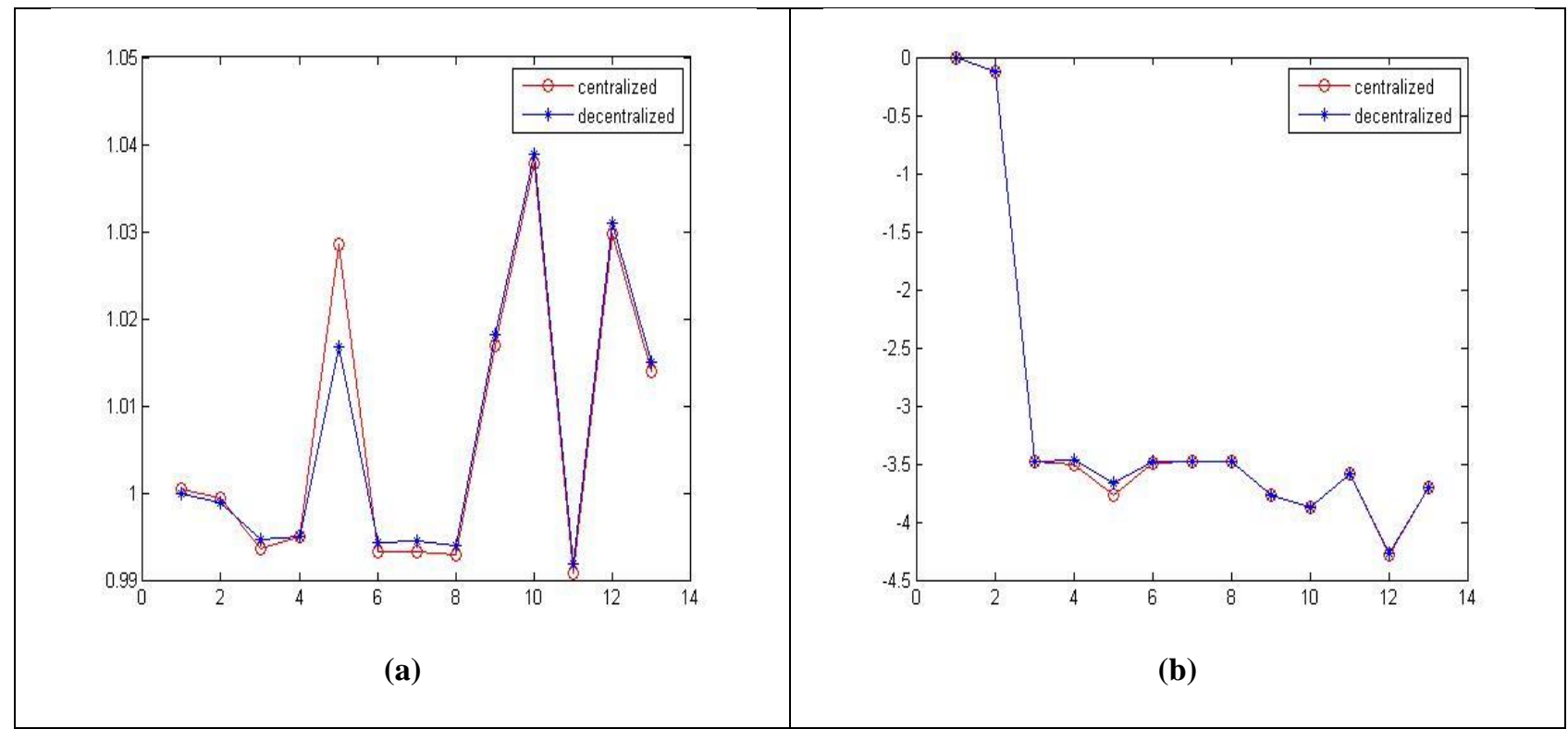

Fig 5.10: (a) Comparison of Voltage magnitudes of proposed method with the centralized solution, (b) Comparison of Voltage angles of proposed method with the centralized solution

From Fig 5.10 it is clearly evident that the proposed algorithm works even in the presence of error in the system.

\subsection{Test Case 2}

The test system for the case study is a $12.66 \mathrm{kV}$ radial distribution system with 33 buses with distributed generation. DG's are 3 small power producers who can provide only firm active power to the system by their DG units. The producers are located at buses 16, 22 and 30 with capacities of 250,250 , and $500 \mathrm{~kW}$, respectively. Its total complex power demand is $3.715+$ j2.305 MVA. Also, the base network power loss is $202.6762 \mathrm{~kW}$. The network configuration is 
shown Fig 5.10 [46]. In this system bus-1 is used as the slack bus. The power flow solution of this system is shown in Table 5.10.

\section{SUBSTATION}

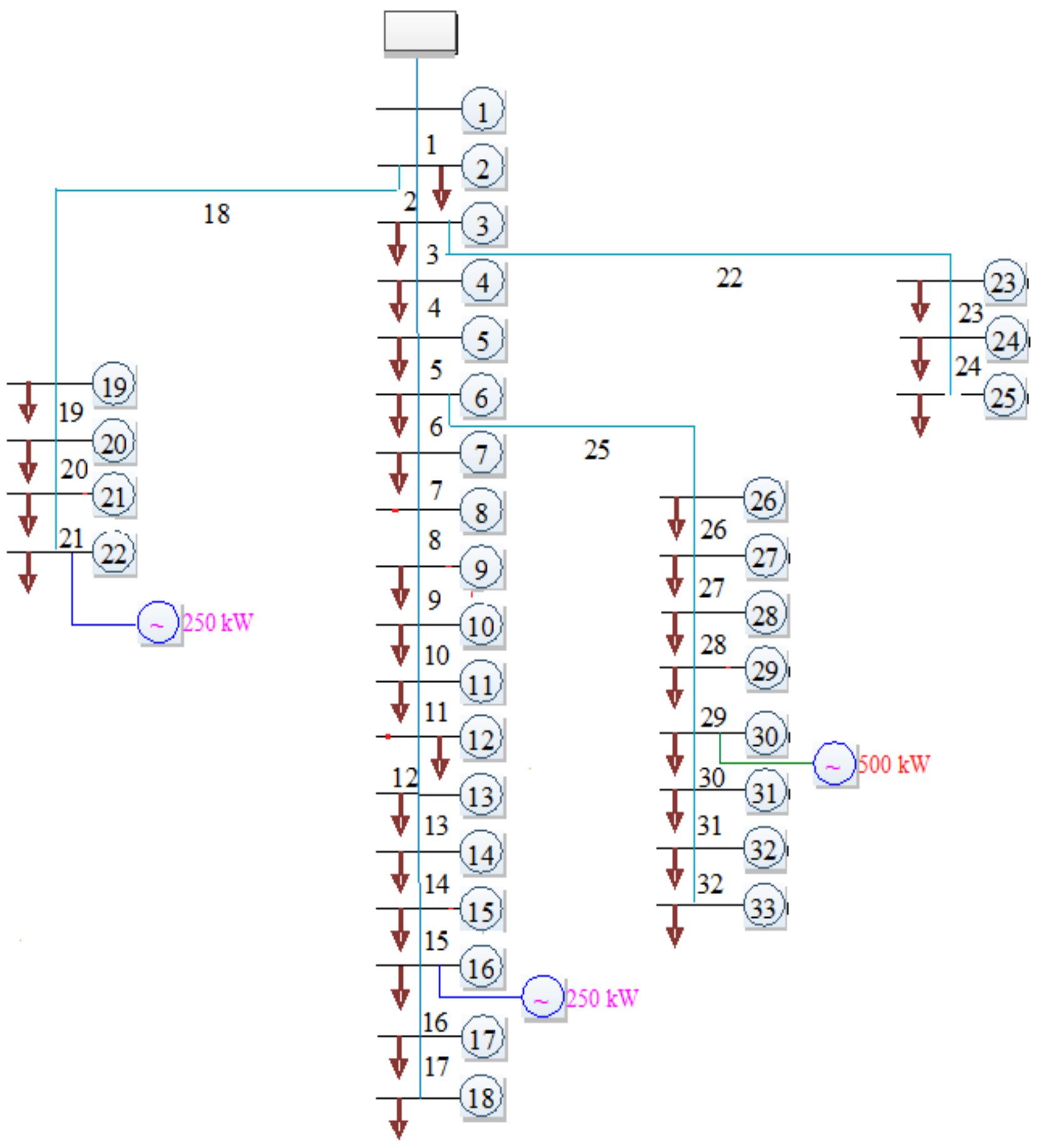

Fig 5.11: Test Case 2 - IEEE 33-bus system 


\begin{tabular}{|c|c|c|c|c|c|c|}
\hline Bus No. & $\begin{array}{c}\text { Voltage } \\
\text { Mag.(p.u) }\end{array}$ & Angle(degree) & MW(Load) & Mvar(Load) & MW(Gen) & Mvar(Gen) \\
\hline 1. & 1.000 & 0.000 & 0.000 & 0.000 & 2.894 & 3.435 \\
\hline 2. & 0.997 & 0.064 & 0.100 & 0.060 & 0.000 & 0.000 \\
\hline 3. & 0.984 & 0.352 & 0.090 & 0.040 & 0.000 & 0.000 \\
\hline 4. & 0.978 & 0.573 & 0.120 & 0.080 & 0.000 & 0.000 \\
\hline 5. & 0.971 & 0.804 & 0.060 & 0.030 & 0.000 & 0.000 \\
\hline 6. & 0.953 & 1.157 & 0.060 & 0.020 & 0.000 & 0.000 \\
\hline 7. & 0.949 & 1.010 & 0.200 & 0.100 & 0.000 & 0.000 \\
\hline 8. & 0.945 & 1.141 & 0.200 & 0.100 & 0.000 & 0.000 \\
\hline 9. & 0.940 & 1.249 & 0.060 & 0.020 & 0.000 & 0.000 \\
\hline 10. & 0.934 & 1.371 & 0.060 & 0.020 & 0.000 & 0.000 \\
\hline 11. & 0.934 & 1.405 & 0.045 & 0.030 & 0.000 & 0.000 \\
\hline 12. & 0.933 & 1.468 & 0.060 & 0.035 & 0.000 & 0.000 \\
\hline 13 & 0.927 & 1.652 & 0.060 & 0.035 & 0.000 & 0.000 \\
\hline 14 & 0.924 & 1.707 & 0.120 & 0.080 & 0.000 & 0.000 \\
\hline 15 & 0.923 & 1.789 & 0.060 & 0.010 & 0.000 & 0.000 \\
\hline 16 & 0.922 & 1.903 & 0.060 & 0.020 & 0.250 & -0.311 \\
\hline 17 & 0.920 & 1.827 & 0.060 & 0.020 & 0.000 & 0.000 \\
\hline 18 & 0.920 & 1.817 & 0.090 & 0.040 & 0.000 & 0.000 \\
\hline 19 & 0.997 & 0.081 & 0.090 & 0.040 & 0.000 & 0.000 \\
\hline 20 & 0.994 & 0.261 & 0.090 & 0.040 & 0.000 & 0.000 \\
\hline 21 & 0.993 & 0.319 & 0.090 & 0.040 & 0.000 & 0.000 \\
\hline 22 & 0.992 & 0.442 & 0.090 & 0.040 & 0.250 & -0.232 \\
\hline 23 & 0.981 & 0.321 & 0.090 & 0.050 & 0.000 & 0.000 \\
\hline 24 & 0.974 & 0.232 & 0.420 & 0.200 & 0.000 & 0.000 \\
\hline 25 & 0.971 & 0.188 & 0.420 & 0.200 & 0.000 & 0.000 \\
\hline 26 & 0.952 & 1.257 & 0.060 & 0.025 & 0.000 & 0.000 \\
\hline 27 & 0.950 & 1.399 & 0.060 & 0.025 & 0.000 & 0.000 \\
\hline 28 & 0.939 & 1.884 & 0.060 & 0.025 & 0.000 & 0.000 \\
\hline 29 & 0.931 & 2.271 & 0.120 & 0.070 & 0.000 & 0.000 \\
\hline 30 & 0.928 & 2.534 & 0.200 & 0.600 & 0.500 & -0.570 \\
\hline 31 & 0.924 & 2.451 & 0.150 & 0.070 & 0.000 & 0.000 \\
\hline 32 & 0.923 & 2.428 & 0.210 & 0.100 & 0.000 & 0.000 \\
\hline 33 & 0.923 & 2.420 & 0.060 & 0.040 & 0.000 & 0.000 \\
\hline Total & & & 3.715 & 2.305 & 3.894 & 2.322 \\
\hline
\end{tabular}

Table 5.13: Power Flow Solution of 33-bus system 
Before dividing the system into areas the states of the entire system (centralized) are first estimated. For this we will be using Conventional WLS technique. This estimation is mainly done to compare the accuracy with the decentralized solution. Table 5.11 gives the details of measurements used and their respective covariance's. Table 5.12 is the solution to the centralized state estimation.

\begin{tabular}{|c|c|}
\hline Measurements & Measurement Error \\
\hline Voltage Magnitude & $9 \mathrm{e}-2$ \\
\hline Real Power Injection & $64 \mathrm{e}-2$ \\
\hline Reactive Power Injection & $64 \mathrm{e}-2$ \\
\hline Real Power Flows & $64 \mathrm{e}-2$ \\
\hline Reactive Power Flows & $64 \mathrm{e}-2$ \\
\hline
\end{tabular}

Table 5.14: Measurements used for 33-bus system

\begin{tabular}{|c|c|c|}
\hline Bus No. & $\begin{array}{c}\text { Voltage } \\
\text { Mag.(p.u) }\end{array}$ & Angle(degree) \\
\hline 1. & 1.0098 & 0.000 \\
\hline 2. & 1.0072 & 0.0631 \\
\hline 3. & 0.9943 & 0.3454 \\
\hline 4. & 0.9877 & 0.5627 \\
\hline 5. & 0.9812 & 0.7895 \\
\hline 6. & 0.9812 & 1.1354 \\
\hline 7. & 0.9590 & 0.9878 \\
\hline 8. & 0.9549 & 1.1190 \\
\hline 9. & 0.9488 & 1.2245 \\
\hline 10. & 0.9433 & 1.3440 \\
\hline 11. & 0.9426 & 1.3782 \\
\hline 12. & 0.9415 & 1.4408 \\
\hline 13. & 0.9353 & 1.6629 \\
\hline 14 & 0.9325 & 1.6748 \\
\hline 15 & 0.9310 & 1.7560 \\
\hline 16 & 0.9297 & 1.8735 \\
\hline 17 & 0.9278 & 1.7875 \\
\hline 18 & 0.9272 & 1.7765 \\
\hline & & \\
\hline
\end{tabular}




\begin{tabular}{|l|l|l|}
\hline 19 & 1.0067 & 0.0799 \\
\hline 20 & 1.0039 & 0.2568 \\
\hline 21 & 1.0032 & 0.3140 \\
\hline 22 & 1.0024 & 0.4358 \\
\hline 23 & 0.9908 & 0.3153 \\
\hline 24 & 0.9842 & 0.2289 \\
\hline 25 & 0.9809 & 0.1850 \\
\hline 26 & 0.9617 & 1.2336 \\
\hline 27 & 0.9596 & 1.3724 \\
\hline 28 & 0.9486 & 1.8484 \\
\hline 29 & 0.9408 & 2.2280 \\
\hline 30 & 0.9381 & 2.4890 \\
\hline 31 & 0.9341 & 2.3994 \\
\hline 32 & 0.9332 & 2.3762 \\
\hline 33 & 0.9330 & 2.3684 \\
\hline
\end{tabular}

Table 5.15: Estimated states of 33-bus system

Now this system is broken into three overlapping areas i.e. it has one bus (agent) common to all the areas. In this system Bus 6 will be used as the agent. Fig.5.11 depicts the division of the 33bus system into two overlapping areas.

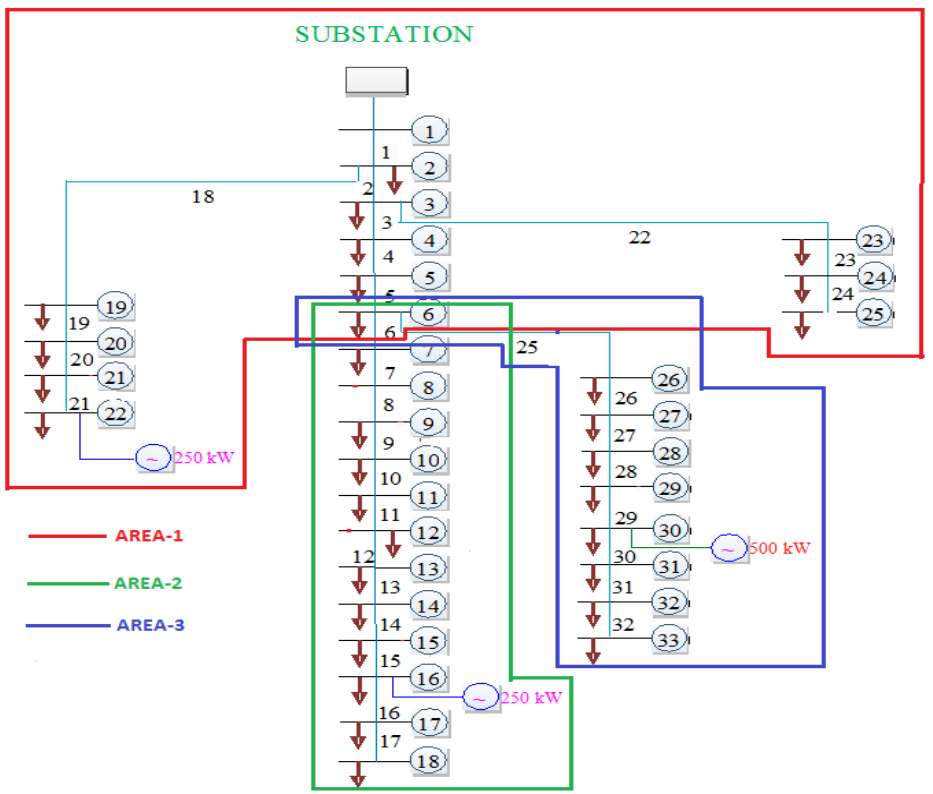

Fig 5.12: 33-Bus System with Three Areas 


\subsubsection{Area 1}

This area consists of 13 buses out of which bus-1 is used as the slack bus and shown in Fig.5.12. As said earlier bus-6 is the agent.

\section{SUBSTATION}

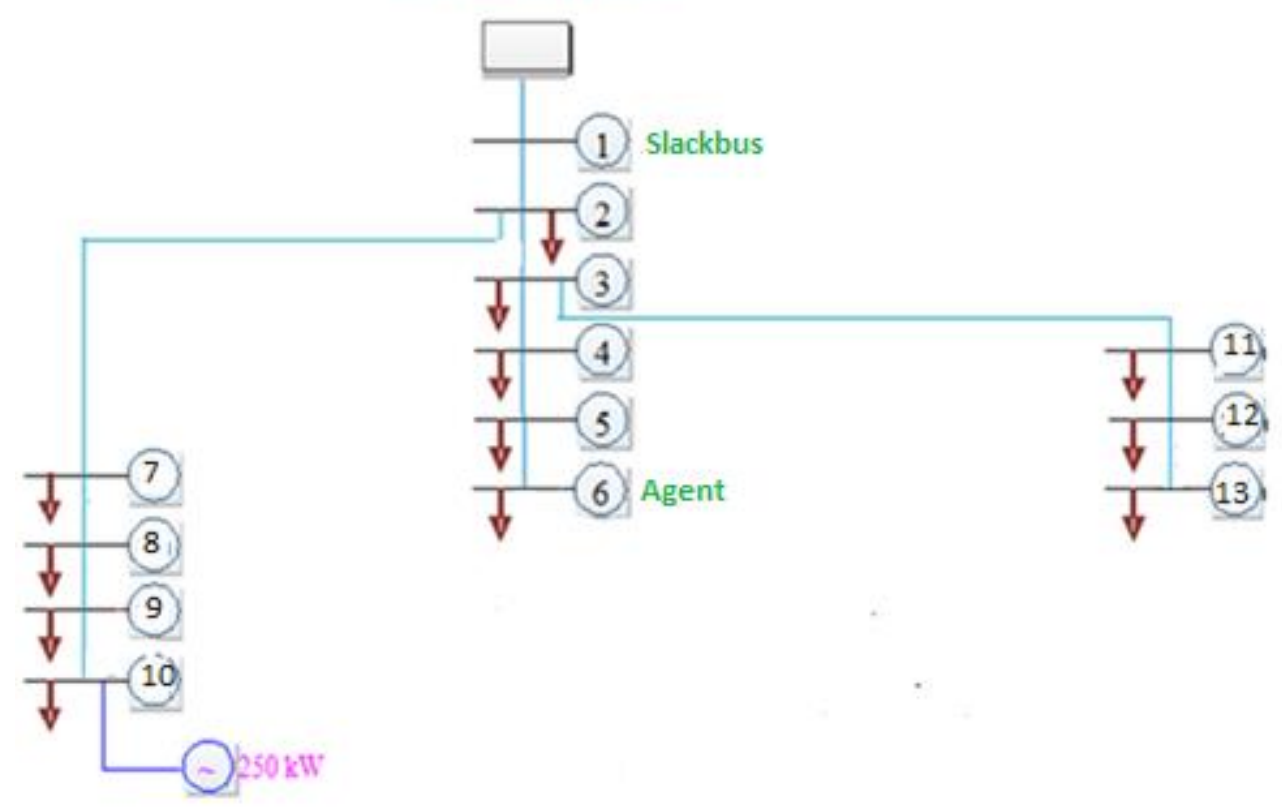

Fig 5.13: Test Case 2: Area-1

Before performing power flow to this new system the agent's (bus-6) load is initialized using the formulas shown below:

$$
\begin{gathered}
\text { Real load } P_{L}(\text { bus }-6)=\sum_{i} P_{L(i)}-\sum_{k} P_{g(k)} \\
\text { Reactive load } Q_{L}(\text { bus }-6)=\sum_{i} Q_{L(i)}-\sum_{k} Q_{g(k)}
\end{gathered}
$$

Where

$i \quad$ - All the load buses in the neighboring areas i.e. Area-2 \& Area-3 in this case.

$k \quad$ - All the generation buses in the neighboring areas i.e. Area- $2 \&$ Area-3 in this case.

$P_{L(i)}$ - Real load at $i_{t h}$ bus.

$Q_{L(i)}$ - Reactive load at $i_{t h}$ bus. 
$P_{g(i)}-$ Real power generation at $k_{t h}$ bus.

$Q_{g(k)}$ - Reactive power generation at $k_{t h}$ bus.

Once we are done with initializing the agent then the power flow is run for this area. The power flow is done using Newton-Raphson method and the solution is shown in Table 5.13.

\begin{tabular}{|c|c|c|c|c|c|c|}
\hline Bus No. & $\begin{array}{c}\text { Voltage } \\
\text { Mag.(p.u) }\end{array}$ & Angle(degree) & MW(Load) & Mvar(Load) & MW(Gen) & Mvar(Gen) \\
\hline 1. & 1.000 & 0.000 & 0.000 & 0.000 & 2.770 & 3.388 \\
\hline 2. & 0.997 & 0.066 & 0.100 & 0.060 & 0.000 & 0.000 \\
\hline 3. & 0.985 & 0.366 & 0.090 & 0.040 & 0.000 & 0.000 \\
\hline 4. & 0.978 & 0.598 & 0.120 & 0.080 & 0.000 & 0.000 \\
\hline 5. & 0.972 & 0.0840 & 0.060 & 0.030 & 0.000 & 0.000 \\
\hline 6. & 0.954 & 1.224 & 1.305 & 2.366 & 0.000 & 0.000 \\
\hline 7. & 0.997 & 0.083 & 0.090 & 0.040 & 0.000 & 0.000 \\
\hline 8. & 0.994 & 0.261 & 0.090 & 0.040 & 0.000 & 0.000 \\
\hline 9. & 0.993 & 0.318 & 0.090 & 0.040 & 0.000 & 0.000 \\
\hline 10. & 0.992 & 0.440 & 0.090 & 0.040 & 0.250 & -0.250 \\
\hline 11. & 0.981 & 0.334 & 0.090 & 0.040 & 0.000 & 0.000 \\
\hline 12. & 0.975 & 0.247 & 0.420 & 0.200 & 0.000 & 0.000 \\
\hline 13. & 0.971 & 0.203 & 0.420 & 0.200 & 0.000 & 0.000 \\
\hline Total & & & 2.965 & 3.176 & 3.020 & 3.139 \\
\hline
\end{tabular}

Table 5.16: Power flow Solution of Area-1

Now before communicating with the other areas the states of this area need to be estimated using WLS technique. The measurements and the measurement error values used here are same as the values used in Table.5.11. The estimated states are shown in Table 5.14.

\begin{tabular}{|c|c|c|}
\hline Bus No. & $\begin{array}{c}\text { Voltage } \\
\text { Mag.(p.u) }\end{array}$ & Angle(degree) \\
\hline 1. & 1.0009 & 0.000 \\
\hline 2. & 0.9983 & 0.0658 \\
\hline
\end{tabular}




\begin{tabular}{|l|l|l|}
\hline 3. & 0.9856 & 0.3641 \\
\hline 4. & 0.9791 & 0.5955 \\
\hline 5. & 0.9728 & 0.8373 \\
\hline 6. & 0.9550 & 1.2240 \\
\hline 7. & 0.9978 & 0.0823 \\
\hline 8. & 0.9947 & 0.2604 \\
\hline 9. & 0.9940 & 0.3183 \\
\hline 10. & 0.9930 & 0.4429 \\
\hline 11. & 0.9822 & 0.3319 \\
\hline 12. & 0.9756 & 0.2448 \\
\hline 13. & 0.9723 & 0.2007 \\
\hline
\end{tabular}

Table 5.17: Estimated states of Area-1 before the communication

These estimated states are then compared with the states obtained from the centralized solution (Table 5.12) and it is assured that only the buses which are present in this area needed to be compared. This is shown in Fig.5.13.

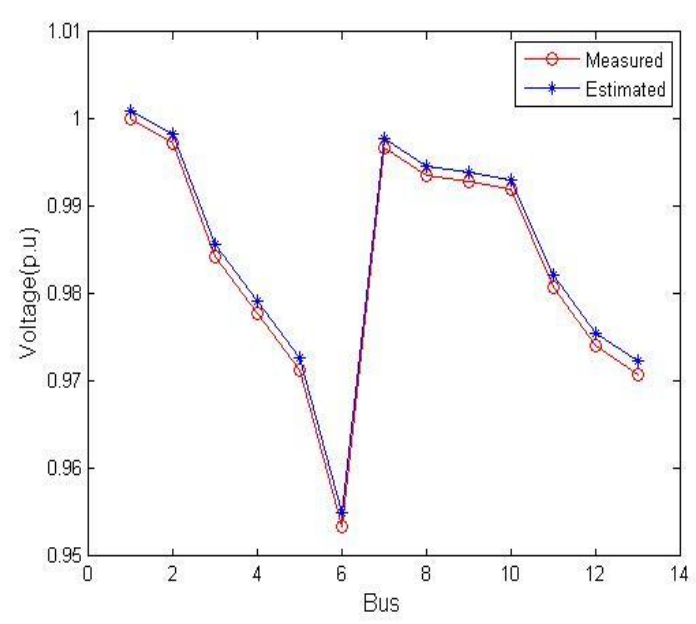

(a)

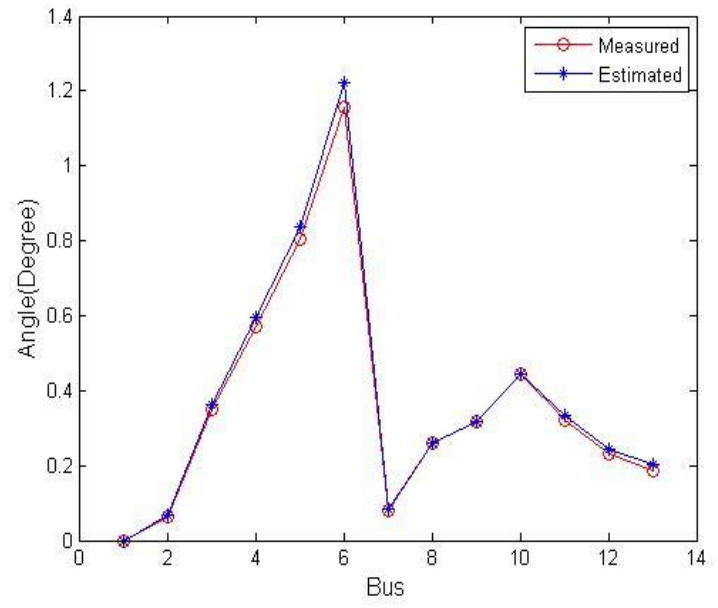

(b)

Fig 5.14: (a) Comparison of Voltage magnitudes of Area-1 with the measured values, (b) Comparison of Voltage angles of Area-1 with the measured values.

When we look at the above plots both the voltage magnitudes and the angles are converged. This is because area-1 has the same slack bus as in original test system (33-bus). So we will be using this point in developing a strategy to communicate between the areas. 


\subsubsection{Area 2}

This area consists of 13 buses out of which bus-1 is used as the slack bus shown in Fig.5.14. In this area bus- 13 is the agent.

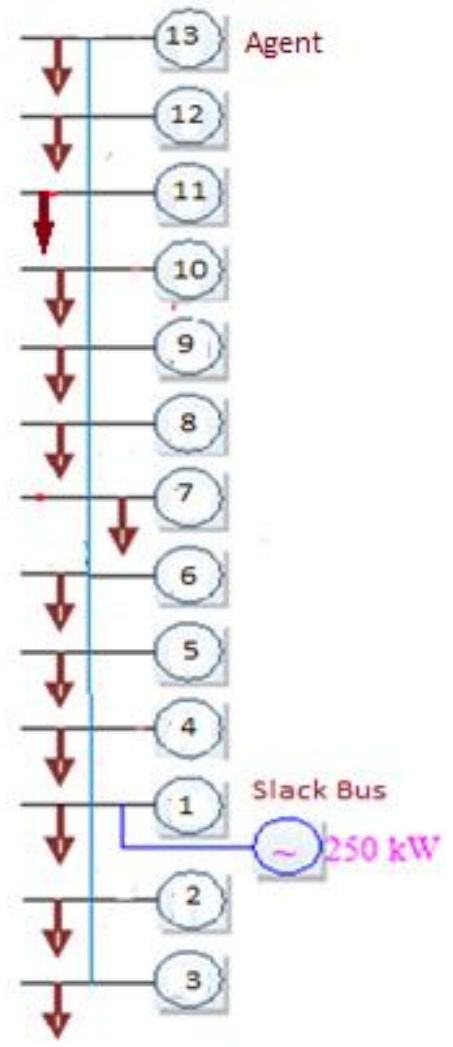

Fig 5.15: Test Case 2: Area-2

Again the agent(bus-13 in this case) is initialized before running the power flow and this is done by using the equations (5.6) and (5.7) where $i$ and $k$ are all the load buses and generation buses respectively of Area-1 and Area-3. The power flow is done using Newton-Raphson method and the solution is shown in Table.5.15.

\begin{tabular}{|c|c|c|c|c|c|c|}
\hline Bus No. & $\begin{array}{c}\text { Voltage } \\
\text { Mag.(p.u) }\end{array}$ & Angle(degree) & MW(Load) & Mvar(Load) & MW(Gen) & Mvar(Gen) \\
\hline 1. & 0.922 & 0.000 & 0.060 & 0.020 & -0.023 & -0.408 \\
\hline 2. & 0.920 & -0.076 & 0.060 & 0.020 & 0.000 & 0.000 \\
\hline 3. & 0.920 & -0.085 & 0.090 & 0.040 & 0.000 & 0.000 \\
\hline 4. & 0.924 & -0.094 & 0.060 & 0.010 & 0.000 & 0.000 \\
\hline
\end{tabular}




\begin{tabular}{|c|c|c|c|c|c|c|}
\hline 5. & 0.926 & -0.154 & 0.120 & 0.080 & 0.000 & 0.000 \\
\hline 6. & 0.930 & -0.174 & 0.060 & 0.035 & 0.000 & 0.000 \\
\hline 7. & 0.937 & -0.313 & 0.060 & 0.035 & 0.000 & 0.000 \\
\hline 8. & 0.939 & -0.375 & 0.045 & 0.030 & 0.000 & 0.000 \\
\hline 9. & 0.940 & -0.409 & 0.060 & 0.020 & 0.000 & 0.000 \\
\hline 10. & 0.946 & -0.504 & 0.060 & 0.020 & 0.000 & 0.000 \\
\hline 11. & 0.953 & -0.586 & 0.200 & 0.100 & 0.000 & 0.000 \\
\hline 12. & 0.958 & -0.715 & 0.200 & 0.100 & 0.000 & 0.000 \\
\hline 13. & 0.963 & -0.536 & -1.004 & -0.838 & 0.000 & 0.000 \\
\hline Total & & & 0.071 & -0.328 & -0.023 & -0.408 \\
\hline
\end{tabular}

Table 5.18: Power flow Solution of Area-2

Now before communicating with the other area the states of this area are first estimated using WLS technique. The measurements and the measurement error values used here are same as the values used in Table.5.11. The estimated states are shown in Table 5.16.

\begin{tabular}{|c|c|c|}
\hline Bus No. & $\begin{array}{c}\text { Voltage } \\
\text { Mag.(p.u) }\end{array}$ & Angle(degree) \\
\hline 1. & 0.9220 & 0.0000 \\
\hline 2. & 0.9206 & -0.0700 \\
\hline 3. & 0.9201 & -0.0801 \\
\hline 4. & 0.9244 & 0.0953 \\
\hline 5. & 0.9266 & -0.1540 \\
\hline 6. & 0.9300 & -0.1731 \\
\hline 7. & 0.9375 & -0.3128 \\
\hline 8. & 0.9390 & -0.3743 \\
\hline 9. & 0.9399 & -0.4082 \\
\hline 10. & 0.9465 & -0.5037 \\
\hline 11. & 0.9535 & -0.5852 \\
\hline 12. & 0.9583 & -0.7143 \\
\hline 13. & 0.9629 & -0.5347 \\
\hline
\end{tabular}

Table 5.19: Estimated states of Area-2 before the communication 


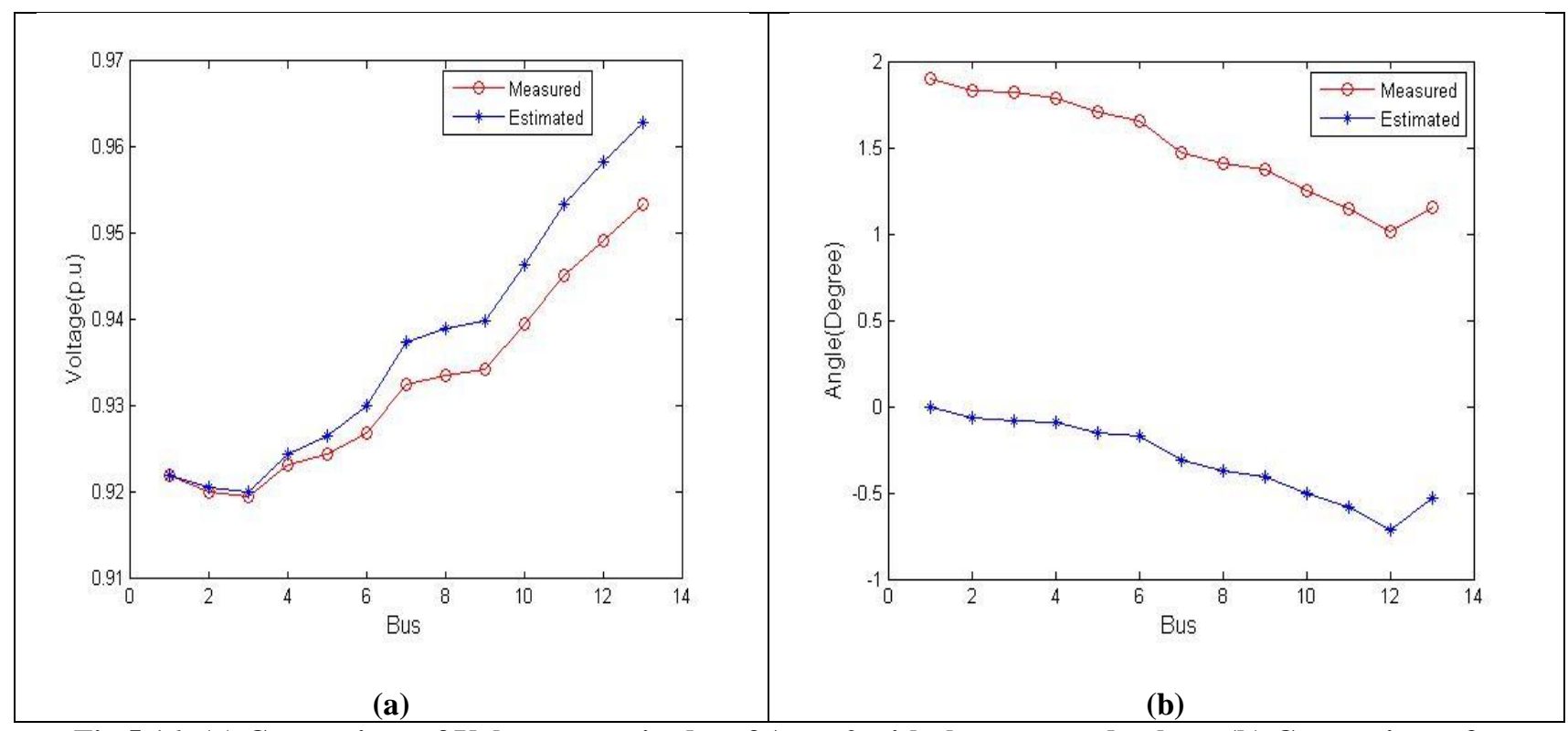

Fig 5.16: (a) Comparison of Voltage magnitudes of Area-2 with the measured values, (b) Comparison of Voltage angles of Area-2 with the measured values.

When we look at the above plots voltage magnitudes are converged but there is a big mismatch in the angles estimated. This can be minimized by communicating with the other areas and this is done once we go through the third area also.

\subsubsection{Area 3}

This area consists of 9 buses out of which bus-1 is used as the slack bus shown in Fig.5.16. In this area bus- 9 is the agent.

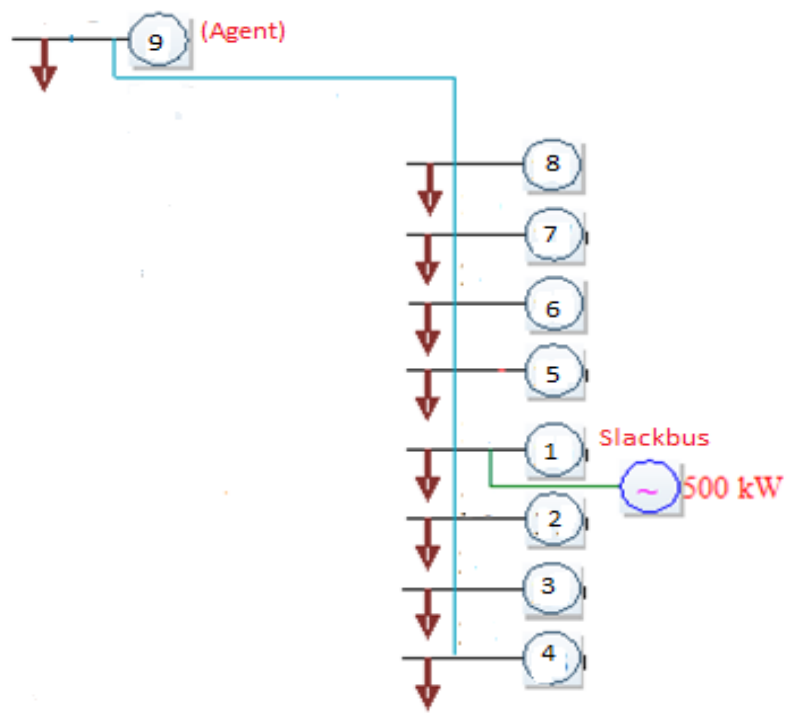

Fig 5.17: Test Case 2: Area-3 
Again the agent (bus-9) is initialized before running the power flow and this is done by using the equations (5.6) and (5.7) where $i$ and $k$ are all the load buses and generation buses respectively of Area-1 and Area-2. The power flow is done using Newton-Raphson method and the solution is shown in Table.5.17.

\begin{tabular}{|c|c|c|c|c|c|c|}
\hline Bus No. & $\begin{array}{c}\text { Voltage } \\
\text { Mag.(p.u) }\end{array}$ & Angle(degree) & MW(Load) & Mvar(Load) & MW(Gen) & Mvar(Gen) \\
\hline 1. & 0.935 & 0.000 & 0.200 & 0.600 & 0.277 & -0.603 \\
\hline 2. & 0.931 & -0.082 & 0.150 & 0.070 & 0.000 & 0.000 \\
\hline 3. & 0.930 & -0.104 & 0.210 & 0.100 & 0.000 & 0.000 \\
\hline 4. & 0.930 & -0.112 & 0.060 & 0.040 & 0.000 & 0.000 \\
\hline 5. & 0.938 & -0.255 & 0.120 & 0.070 & 0.000 & 0.000 \\
\hline 6. & 0.947 & -0.611 & 0.060 & 0.025 & 0.000 & 0.000 \\
\hline 7. & 0.959 & -1.057 & 0.060 & 0.025 & 0.000 & 0.000 \\
\hline 8. & 0.962 & -1.194 & 0.060 & 0.025 & 0.000 & 0.000 \\
\hline 9. & 0.964 & -1.290 & -0.599 & -1.542 & 0.000 & 0.000 \\
\hline Total & & & 0.321 & -0.587 & 0.277 & -0.603 \\
\hline
\end{tabular}

Table 5.20: Power flow Solution of Area-3

Now before communicating with the other area the states of this area are first estimated using WLS technique the states are estimated. The measurements and the measurement error values used here are same as the values used in Table.5.10. The estimated states are shown in Table 5.18 .

\begin{tabular}{|c|c|c|}
\hline Bus No. & $\begin{array}{c}\text { Voltage } \\
\text { Mag.(p.u) }\end{array}$ & Angle(degree) \\
\hline 1. & 0.9350 & 0.000 \\
\hline 2. & 0.9312 & -0.0791 \\
\hline 3. & 0.9303 & -0.1009 \\
\hline 4. & 0.9300 & -0.1080 \\
\hline 5. & 0.9384 & -0.2547 \\
\hline 6. & 0.9472 & -0.6097 \\
\hline 7. & 0.9595 & -1.0547 \\
\hline
\end{tabular}




\begin{tabular}{|l|l|l|}
\hline 8. & 0.9619 & -1.1912 \\
\hline 9. & 0.9637 & -1.2880 \\
\hline
\end{tabular}

Table 5.21: Estimated states of Area-3 before the communication.

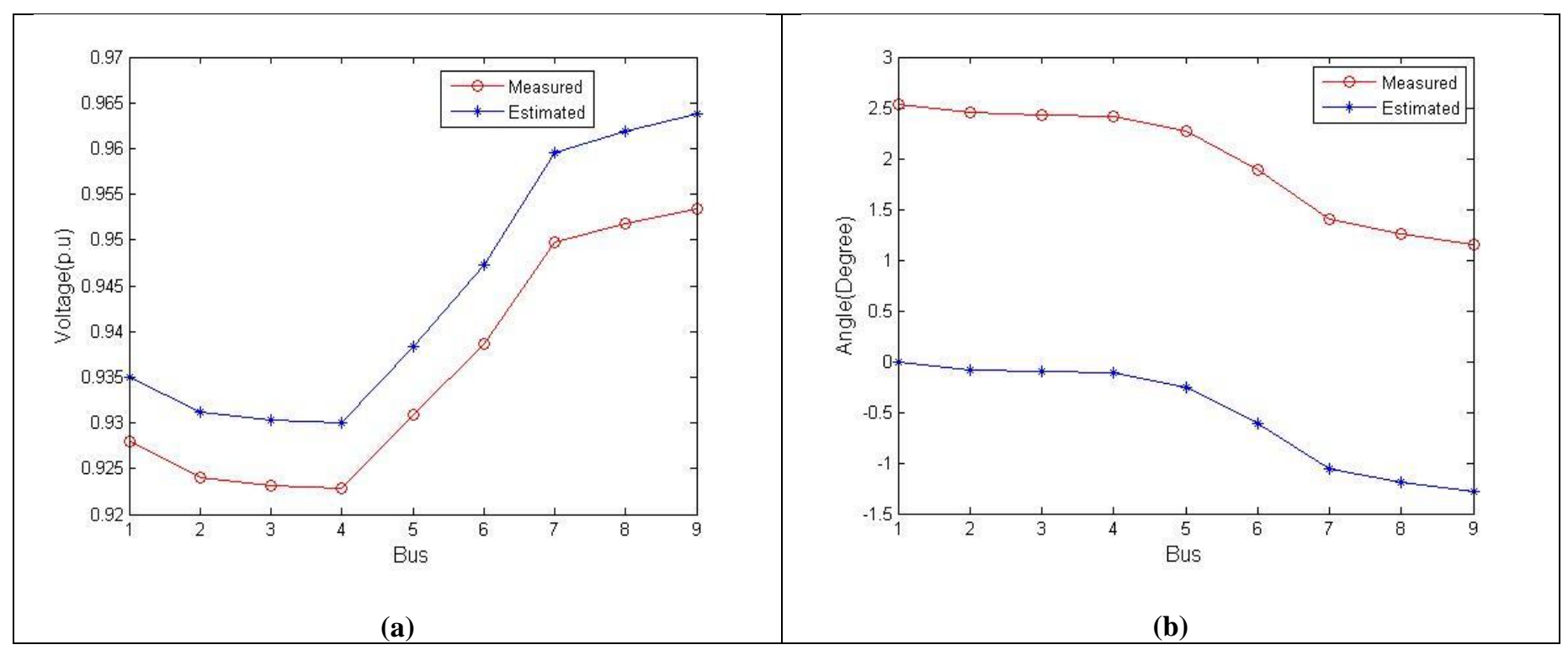

Fig 5.18: (a) Comparison of Voltage magnitudes of Area-3 with the measured values, (b) Comparison of Voltage angles of Area-3 with the measured values.

When we look at the above plots voltage magnitudes are converged but there is a big mismatch in the angles estimated and as said earlier this can be minimized by communicating with the other areas.

If we look at the three areas bus-6, bus-13 and bus-9 in Area-1, Area-2 and Area-3 are used as the agents i.e. these buses are one and the same. So the states of all the three buses should be equal to one another rather should be equal to bus- 6 of Area-1 since the estimated states of Area1 are already converged. Using this criteria, communication is done between the areas and the difference in the agent's angle of Area-1 with the other two areas separately is calculated. This difference in angles is added to all the bus angles including the agent present in the neighboring areas i.e. Area-2 and Area-3 in this case.

For Area-2

$$
\Delta \text { Angle }=1.2240-(-0.5347)=1.7587
$$


This value of $\Delta$ Angle is added to all the bus angles present in area-2 through which consensus will be achieved. The new updated states in area-2 are shown in Table 5.19.

\begin{tabular}{|c|c|c|}
\hline Bus No. & $\begin{array}{c}\text { Voltage } \\
\text { Mag.(p.u) }\end{array}$ & Angle(degree) \\
\hline 1. & 0.9220 & 1.7587 \\
\hline 2. & 0.9206 & 1.6887 \\
\hline 3. & 0.9201 & 1.6786 \\
\hline 4. & 0.9244 & 1.6634 \\
\hline 5. & 0.9266 & 1.6047 \\
\hline 6. & 0.9300 & 1.5857 \\
\hline 7. & 0.9375 & 1.4459 \\
\hline 8. & 0.9390 & 1.3844 \\
\hline 9. & 0.9399 & 1.3505 \\
\hline 10. & 0.9465 & 1.2550 \\
\hline 11. & 0.9535 & 1.1736 \\
\hline 12. & 0.9583 & 1.0444 \\
\hline 13 & 0.9629 & 1.2240 \\
\hline
\end{tabular}

Table 5.22: Updated States of Area-2

For Area-3

$$
\Delta \text { Angle }=1.2240-(-1.2880)=2.5120
$$

This value of $\Delta$ Angle is added to all the bus angles present in area- 3 through which consensus will be achieved. The new updated states in area-3 are shown in Table 5.20.

\begin{tabular}{|c|c|c|}
\hline Bus No. & $\begin{array}{c}\text { Voltage } \\
\text { Mag.(p.u) }\end{array}$ & Angle(degree) \\
\hline 1. & 0.9350 & 2.5120 \\
\hline 2. & 0.9312 & 2.4328 \\
\hline 3. & 0.9303 & 2.4110 \\
\hline 4. & 0.9300 & 2.4309 \\
\hline 5. & 0.9384 & 2.2572 \\
\hline 6. & 0.9472 & 1.9022 \\
\hline 7. & 0.9595 & 1.4572 \\
\hline 8. & 0.9619 & 1.3207 \\
\hline
\end{tabular}




\begin{tabular}{|l|l|l|}
\hline 9. & 0.9637 & 1.2240 \\
\hline
\end{tabular}

Table 5.23: Updated States of Area-3

Therefore combining the states from all the three local estimators (i.e. from Table 5.14, table 5.19 and Table 5.20) the final decentralized solution is obtained which is tabulated in Table 5.21 .

\begin{tabular}{|c|c|c|}
\hline Bus No. & $\begin{array}{c}\text { Voltage } \\
\text { Mag.(p.u) }\end{array}$ & Angle(degree) \\
\hline 1. & 1.0009 & 0.000 \\
\hline 2. & 0.9983 & 0.0658 \\
\hline 3. & 0.9856 & 0.3641 \\
\hline 4. & 0.9791 & 0.5955 \\
\hline 5. & 0.9728 & 0.8373 \\
\hline 6. & 0.9550 & 1.2240 \\
\hline 7. & 0.9583 & 1.0444 \\
\hline 8. & 0.9535 & 1.1736 \\
\hline 9. & 0.9465 & 1.2550 \\
\hline 10 & 0.9399 & 1.3505 \\
\hline 11. & 0.9390 & 1.3844 \\
\hline 12. & 0.9375 & 1.4459 \\
\hline 13. & 0.9300 & 1.5857 \\
\hline 14. & 0.9266 & 1.6047 \\
\hline 15 & 0.9244 & 1.6634 \\
\hline 16 & 0.9220 & 1.7587 \\
\hline 17 & 0.9206 & 1.6887 \\
\hline 18 & 0.9201 & 1.6786 \\
\hline 19 & 0.9978 & 0.0823 \\
\hline 20 & 0.9947 & 0.2604 \\
\hline 21 & 0.9940 & 0.3183 \\
\hline 22 & 0.9930 & 0.4429 \\
\hline 23 & 0.9822 & 0.3319 \\
\hline 24 & 0.9756 & 0.2448 \\
\hline 25 & 0.9723 & 0.2007 \\
\hline 26 & 0.9619 & 1.3207 \\
\hline 27 & 0.9595 & 1.4572 \\
\hline 28 & 0.9472 & 1.9022 \\
\hline
\end{tabular}




\begin{tabular}{|l|l|l|}
\hline 29 & 0.9384 & 2.2572 \\
\hline 30 & 0.9350 & 2.5120 \\
\hline 31 & 0.9312 & 2.4328 \\
\hline 32 & 0.9303 & 2.4110 \\
\hline 33 & 0.9300 & 2.4309 \\
\hline
\end{tabular}

Table 5.24 : Proposed Solution for Test Case-2

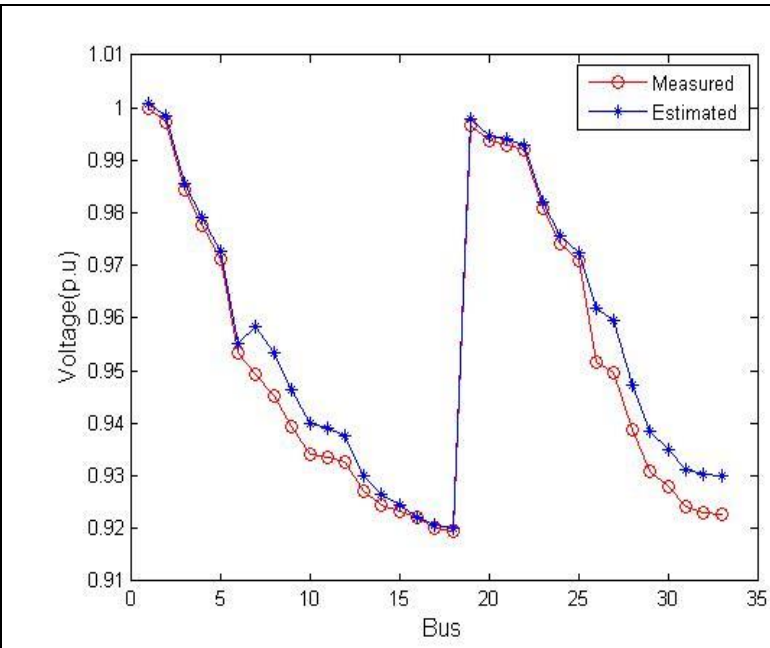

(a)

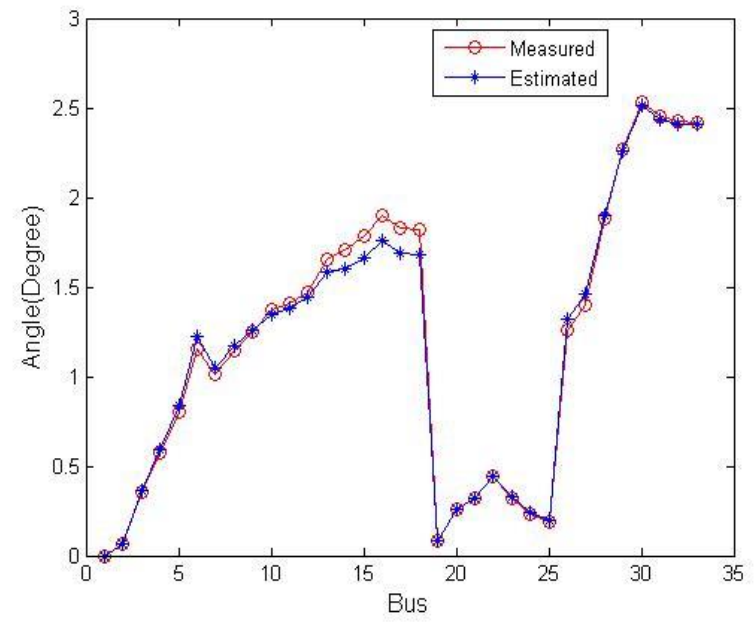

(b)

Fig 5.19: (a) Comparison of Voltage magnitudes of proposed solution with the estimated values, (b) Comparison of Voltage angles of proposed solution with the estimated values.

From Fig 5.9 and Fig 5.18 we can clearly see that both estimated and measured values are nearly equal and the error between the values is very less. This shows that the consensus is achieved between the local estimators and therefore the proposed algorithm worked successfully on both the test systems. 


\section{CONCLUSION AND FUTURE WORK}

The work described in this thesis is concerned with the development of decentralized method for state estimation in power systems. It has been shown that the developed method is practical for current power systems and these methods have also been demonstrated on a benchmark power system model. The work also has a wide scope for future research and extensions. The conclusions of this thesis and recommendations for future work are presented as follows.

\subsection{Thesis Conclusions}

Decentralized power system state estimation has been treated here in a unified and systematic manner. The work done in this thesis is summarized as follows:

1. Proposed a distributed state model and a consensus based static state estimation method for smart distribution grid. We specially consider the case when for each agent, the local measurement model is underdetermined and all state elements for a particular agent is completely shared with its neighbors.

2. Global consensus algorithm is employed such that first allowing the local estimators in each area to converge to the desired tolerance, and then apply the coupling constraint corrections without any further local estimation iterations. This asynchronous method of updating the state variables in neighboring areas does not influence the local estimation iterative process and can be incorporated any time after the convergence of the local processes.

3. Simulation results on a radial distribution grid show that the proposed method can give satisfactory convergence based on the appropriate selection of agents.

4. The advantages of the proposed method are as follows: a) high computational efficiency, b) accuracy is similar to the integrated solution, c) highly robust to deal with topology changes, d) bad data processing for buses located close to boundary buses, and e) low data exchange between areas. 


\subsection{Future Work}

In this thesis the proposed method concentrates on the balanced distribution networks so in the future we can try to implement on the unbalanced networks also. In the future, probabilistic model of communication failure will be incorporated to investigate its effect on the performance of state estimation. The proposed algorithm is a static state estimation procedure and based on the performance of the developed method, formulation of a consensus based dynamic state estimation procedure for smart distribution grid is our future objective. The decentralized algorithm presented in this thesis relies on conventional measurements(such as power flows, power injections) for the estimation process so the extension to this work can be incorporation of PMU'S in the system and using PMU measurements for the estimation process. 


\section{REFERENCES}

1. Test guidelines for synchronous unit dynamic testing and model validation," Western Systems Coordinating Council, Tech. Rep., 1997".

2. Generating unit model validation policy," Western Electricity Coordinating Council, Tech. Rep., 2006".

3. A.Abur and A.G.Exposito, Power System State Estimation - Theory and Implementation. New York: Marcel Dekker, 2004.

4. F. F. Wu, "Power system state estimation: a survey," International Journal of Electrical Power \&amp; Energy Systems, vol. 12, pp. 80-87, 1990.

5. F. Schweppe and D. Rom, "Power system static-state estimation: Parts I, II, \& III," Power Apparatus \& Sys. IEEE Trans., vol. 89, no. 1, pp. 125-130, Jan 1970.

6. A. Gomez-Exposito, A. Abur, A. de la Villa Jaen, and C. Gomez-Quiles, "A Multilevel State Estimation Paradigm for Smart Grids," Proceedings of the IEEE, vol. 99, pp. 952976, 2011.

7. A. Gómez-Expósito, A. de la Villa Jaén, C. Gómez-Quiles, P. Rousseaux, and T. Van Cutsem, "A taxonomy of multi-area state estimation methods," Electric Power Systems Research, vol. 81, pp. 1060-1069, 2011.

8. A. Monticelli, "Electric power system state estimation," Proceedings of the IEEE, vol. 88, no. 2, pp. 262-282, Feb 2000.

9. H. M. Merrill and F. C. Schweppe, "Bad Data Suppression in Power System Static State Estimation," IEEE Trans. Power App. Syst., vol. PAS-90, pp. 2718-2725, 1971.

10. H. P. Horisberger, J. C. Richard, and C. Rossier, "A fast decoupled static state-estimator for electric power systems," IEEE Trans. Power App. Syst., vol. 95, pp. 208-215, 1976.

11. Z. Shan and A. Abur, "Auto tuning of measurement weights in WLS state estimation," IEEE Trans. Power Syst., vol. 19, pp. 2006-2013, 2004.

12. A. Simoes-Costa and V. H. Quintana, "A Robust Numerical Technique for Power System State Estimation," IEEE Trans. Power App. Syst., vol. PAS-100, pp. 691-698, 1981.

13. A. Pandian, K. Parthasarathy, and S. A. Soman, "Towards faster Givens rotations based power system state estimator," IEEE Trans. Power Syst., vol. 14, pp. 837-843, 1999.

14. A. F., P. N., and A. E., "State Estimation with Equality Constraints," presented at the 10th PICA Conference Proceedings, Toronto, 1977. 
15. G. N. Korres, "A Robust Algorithm for Power System State Estimation With Equality Constraints," IEEE Trans. Power Syst., vol. 25, pp. 1531-1541, 2010.

16. E. Handschin, F. C. Schweppe, J. Kohlas, and A. Fiechter, "Bad data analysis for power system state estimation," IEEE Trans. Power App. Syst., vol. 94, pp. 329-337, 1975.

17. E. Caro, A. J. Conejo, R. Minguez, M. Zima, and G. Andersson, "Multiple Bad Data Identification Considering Measurement Dependencies," IEEE Trans. Power Syst., vol. 26, pp. 1953-1961, 2011.

18. N.-d. Xiang, S.-y. Wang, and E.-k. Yu, "A New Approach for Detection and Identification of Multiple Bad Data in Power System State Estimation," IEEE Trans. Power App. Syst., vol. PAS-101, pp. 454-462, 1982.

19. T. Van Cutsem, M. Ribbens-Pavella, and L. Mili, "Hypothesis Testing Identification: A New Method For Bad Data Analysis In Power System State Estimation," IEEE Trans. Power App. Syst., vol. PAS-103, pp. 3239-3252, 1984.

20. M. R. Irving, R. C. Owen, and M. J. H. Sterling, "Power-system state estimation using linear programming," Electrical Engineers, Proceedings of the Institution of, vol. 125, pp. 879-885, 1978.

21. C. K. Gharban and B. J. Cory, "Non-Linear Dynamic Power System State Estimation," IEEE Trans. Power Syst., vol. 1, pp. 276-283, 1986.

22. K. K. C. Yu and N. R. Watson, "An Approximate Method for Transient State Estimation," IEEE Trans. Power Del., vol. 22, pp. 1680-1687, 2007.

23. P. Ashton, G. A. Taylor, M. R. Irving, I. Pisica, A. Carter, and M. E. Bradley, "Novel Application of Detrended Fluctuation Analysis for State Estimation Using Synchrophasor Measurements," IEEE Trans. Power Syst., vol. 28, pp. 1930-1938, 2013.

24. T. Van Cutsem and M. Ribbens-Pavella, "Critical Survey of Hierarchical Methods for State Estimation of Electric Power Systems," IEEE Trans. Power App. Syst., vol. PAS102, pp. 3415-3424, 1983.

25. K.A. Clements, O.J. Denison, and R. J. Ringlee, "A multi-area approach to state estimation in power system networks," presented at the IEEE PES Summer Meeting, San Francisco, 1972.

26. M. S. Kurzyn, "Real-Time State Estimation for Large-Scale Power Systems," IEEE Trans. Power App. Syst., vol. PAS-102, pp. 2055-2063, 1983. 
27. A. Gomez-Exposito and A. de la Villa Jaen, "Two-Level State Estimation With Local Measurement Pre-Processing," IEEE Trans. Power Syst., vol. 24, pp. 676-684, 2009.

28. C. W. Brice and R. K. Cavin, "Multiprocessor Static State Estimation," IEEE Trans. Power App. Syst., vol. PAS-101, pp. 302-308, 1982.

29. S. Y. Lin and C. H. Lin, "An implementable distributed state estimator and distributed bad data processing schemes for electric power systems," IEEE Trans. Power Syst., vol. 9, pp. 1277-1284, 1994.

30. A. J. Conejo, S. de la Torre, and M. Canas, "An Optimization Approach to Multiarea State Estimation," IEEE Trans. Power Syst., vol. 22, pp. 213-221, 2007.

31. Barry Hayes and Milan Prodanovi'c State Estimation Techniques for Electric Power Distribution Systems IMDEA Energy Institute, Madrid, Spain.

32. R. Singh, B. Pal, and R. Jabr, "Choice of estimator for distribution system state estimation,” Gen., Tran. \& Dist., IET, vol. 3, no. 7, pp. 666-678, 2009.

33. M. Baran and A. Kelley, "A branch-current-based state estimation method for distribution systems," Power Sys., IEEE Trans., vol. 10, no. 1, pp. 483-491, Feb 1995.

34. W.-M. Lin, J.-H. Teng, and S.-J. Chen, "A highly efficient algorithm in treating current measurements for the branch-current-based distribution state estimation," Power Delivery, IEEE Trans., vol. 16, no. 3, pp. 433-439, Jul 2001.

35. M. Baran and A. Kelley, "State estimation for real-time monitoring of distribution systems," Power Sys., IEEE Trans., vol. 9, no. 3, pp. 1601-1609, Aug 1994.

36. A. Leite da Silva, M. Do Coutto Filho, and J. F. De Queiroz, "State forecasting in electric power systems," Gen., Tran. \& Dist., IEE Proc., vol. 130, no. 5, pp. 237-244, September 1983.

37. S. Julier and J. Uhlmann, "Unscented filtering and nonlinear estimation," Proceedings of the IEEE, vol. 92, no. 3, pp. 401-422, Mar 2004.

38. A. G'omez-Exp'osito, A. de la Villa Ja'en, C. G'omez-Quiles, P. Rousseaux, and T. V. Cutsem, "A taxonomy of multi-area state estimation methods," Elec. Power Sys. Research, vol. 81, no. 4, pp. 1060 - 1069, 2011.

39. A. Meliopoulos, E. Polymeneas, Z. Tan, R. Huang, and D. Zhao, "Advanced distribution management system,” Smart Grid, IEEE Trans., vol. 4, no. 4, pp. 2109-2117, Dec 2013. 
40. F. F. Wu, "Power system state estimation: a survey," Int. Journal of Elec. Power \& Energy Sys., vol. 12, no. 2, pp. $80-87,1990$.

41. IEEE Standard 399- 1990, "IEEE Recommended Practice for Industrial and Commercial Power System Analysis", IEEE, New York, 1990.

42. IEEE Transactions on Power Delivery, Vol. 14, No. 2, April 1999 "Test Systems for Harmonics Modeling and Simulation".

43. Alam, S.M.S.; Natarajan, B.; Pahwa, A.; Curto, S., "Agent based State Estimation in Smart Distribution Grid," Latin America Transactions, IEEE (Revista IEEE America Latina), vol.13, no.2, pp.496,502, Feb. 2015

44. Y.-F. Huang, S. Werner, J. Huang, N. Kashyap, and V. Gupta, "State estimation in electric power grids: Meeting new challenges presented by the requirements of the future grid,” Signal Processing Magazine, IEEE, vol. 29, no. 5, pp. 33-43, 2012.

45. G. Korres, "A distributed multi area state estimation," Power Systems, IEEE Transactions on, vol. 26, no. 1, pp. 73-84, 2011.

46. Seyed Abbas Taher, Mohammad Hossein Karimi, " Optimal reconfiguration and DG allocation in balanced and unbalanced distribution systems", Ain Shams Engineering Journal (2014) 5, 735-749. 